\title{
Melatonin and its-Remodeled Fecal Microbiota Improve Gut Health Through Inhibiting Oxidative Stress, Autophagy and Inflammation
}

\section{Yingqian Wang}

Northwest A\&F University

\section{Weike Shaoyong}

Northwest A\&F University

Rui Cai

Northwest A\&F University

Xudong Yi

Northwest A\&F University

Wenlong Yong

Northwest A\&F University

Chenyang Wang

Northwest A\&F University

Zhongyun Kou

Northwest A\&F University

Lei Gao

Northwest A\&F University

Guiyan Chu

Northwest A\&F University

Jiangwei Wu

Northwest A\&F University

Weijun Pang ( $\triangle$ pwj1226@nwafu.edu.cn )

Northwest A\&F University https://orcid.org/0000-0003-1740-6536

\section{Research}

Keywords: Melatonin, Fecal microbiota transplantation, Foster care, Gut health, Antioxidation

Posted Date: November 15th, 2021

DOI: https://doi.org/10.21203/rs.3.rs-1049518/v1 
License: (c) (i) This work is licensed under a Creative Commons Attribution 4.0 International License. Read Full License 


\section{Abstract \\ Background}

Gut health is involved in the nutrition absorption, reproduction and lactation in antenatal, perinatal and early weaned mammals. Recent literatures have demonstrated that melatonin functions in aging, cancer and obesity, but to date, few investigations toward exploring whether melatonin-reprogrammed fecal microbiota transplantation (FMT) and foster care (FC) affect gut health have been performed.

\section{Results}

Here, compared with the control group, melatonin and FMT increased intestinal villus height/crypt depth (V/C), inhibited gut oxidative stress, autophagy and inflammation in antenatal and perinatal rats. Likewise, not only melatonin and FMT, but also FC enhanced intestinal V/C through above parallel ways with decreasing intestinal permeability in early weaned rats. Mechanically, melatonin directly strengthened antioxidation, attenuated autophagy and apoptosis in $\mathrm{H}_{2} \mathrm{O}_{2}$-induced IEC6 intestinal epithelial cells. Furthermore, melatonin, FMT and FC reprogrammed intestinal microbiota in which more beneficial microbiota, including Allobaculum, Bifidobacterium and Faecalibaculum produced more metabolic short-chain fatty acids (SCFAs) including acetic acid and butyric acid to protect gut health. Most interestingly, compared with the control group, early weaned rats may get above probiotics via eating or licking the dung of the co-cage rats treated with melatonin in the FC group.

\section{Conclusions}

Overall, melatonin, FMT and FC improved gut health and the potential regulatory mechanism was associated with strengthening antioxidation, suppressing autophagy, inflammatory and apoptosis, as well as producing more SCFAs from reprogrammed gut microbiota. These findings suggest that melatonin, FMT and FC may be novel and effective methods to ameliorate gut health in antenatal, perinatal and weaned mammals.

\section{Background}

The gut health which directly determines nutrition absorption is vital to animal growth and development [1]. There is a high incidence of intestinal diseases worldwide, because characteristics of these diseases are long course chronicity and recurrent attacks which make them more difficult to treat. Therefore, intestinal diseases have been surpassing some chronic diseases, including diabetes and cardiovascular diseases, and has become the most important global public health concern today. Gut microbiota plays a central role in the pathogenesis of these diseases as it mediates environmental changes to the intestinal immune system [2]. Further exploration of the composition and function of the gut microbiota and the potential for its manipulation to cure disease is an area of rapid growth and great promise. Therefore, gut 
microbiota is implicated in mammalian gut health and remodeling healthy intestinal microbiota is beneficial to sick animal in gut microbiota dysbiosis.

The mammalian gut microbiota is composed of trillions of microbes that especially facilitate host gut health and prevent diarrhea, inflammatory bowel disease and irritable bowel syndrome $[3,4]$. The gut microbiota is now considered an important partner of human and animal cells, interacting with virtually all cells [2]. In fact, there are numerous metabolites including short-chain fatty acids (SCFAs), secondary bile acids, indoleacrylic acid, skatole, tryptamine and histamine produced by gut microbiome that can influence mammal metabolism [5-9]. Increasing literatures indicates that the changes in gut microbiota composition are associated with intestinal diseases, obesity and its associated metabolic disorders [1012]. Both baseline microbiota and microbial richness were inversely associated with insulin resistance and diarrhea $[3,13]$. Interestingly, fecal microbiota transplantation (FMT) is an important method for establishing or remodeling functional gut microbiota to improve gut health or cure diseases, such as diarrhea and obesity $[3,14]$. Moreover, the early weaned animals maintained intestinal health possibly through reprogrammed gut microbiota via eating or licking the dung from healthy adult animals. Therefore, foster care (FC) or co-cage feeding may be a novel promising method for establishing or reprogramming functional gut microbiota to ameliorate intestinal health.

The use of probiotics and prebiotics which can replace antibotics is increasing in popularity for both the prevention and treatment of a variety of gut diseases [15]. Within this space, there is much to learn, and novel gut microbial therapeutics are likely to rapidly evolve over the next decade. Based on above analysis, finding probiotics and prebiotics alternatives to prevent gastrointestinal disorders is crucial for gut health in animal and human. Melatonin, as a high effective antioxidant, is the most versatile and ubiquitous hormonal molecule produced not only in the pineal gland, but also in various other tissues of mammal, particularly in the gastrointestinal tract [16]. Moreover, it plays a vital role in the regulation of other hormones to maintain the circadian rhythm, and prevent obesity, neurodegenerative diseases and viral infection [17-20]. Recently, increasing literatures have shown that melatonin is implicated in antioxidation, autophagy and inflammation which drives the gut microbiota $[14,21,22]$. However, few clinical or experimental studies evaluated the effects and regulatory mechanism of melatonin, itsremodeled FMT and FC on gut health in antenatal, perinatal and early weaned animals.

Herein, we investigated the effect and underlying mechanism of melatonin, FMT and FC on gut health in antenatal, perinatal female rats and early weaned rats. Our results indicated that melatonin, FMT and FC ameliorated gut health and revealed that the potential regulatory mechanism was associated with enhancing antioxidation and suppressing autophagy, inflammatory and apoptosis, as well as producing more SCFAs from reprogramming gut microbiota. These findings suggested that not only melatonin and its-remodeled FMT, but also FC could be new effective methods for gut disorders caused by oxidative stress to improve mammalian intestinal health.

\section{Results}


The intestinal health of antenatal and perinatal animals and their cubs is implicated in reproduction, lactation and mortality rate. However, few clinical or experimental studies evaluated the effects and regulatory mechanism of melatonin, its-remodeled fecal microbiota and FC or co-cage feeding on mammalian gut health. For this reason, we systematically investigated the effect and underlying mechanism of melatonin, FMT and FC on gut health in antenatal, perinatal female rats and early weaned rats. In this study, the results were divided into four parts: (1) melatonin and its-remodeled FMT improve gut health in antenatal and perinatal rats; (2) melatonin, FMT and FC ameliorate gut health in early weaned rats; (3) melatonin attenuates oxidative stress, autophagy and apoptosis in $\mathrm{H}_{2} \mathrm{O}_{2}$-induced rat intestinal epithelial cell 6 (IEC-6); (4) melatonin, FMT and FC improve gut health by increasing short-chain fatty acids (SCFAs) produced by remodel gut microbiota mainly including Allobaculum, Bifidobacterium and Faecalibaculum.

\section{Part 1. Melatonin and FMT improve gut health in antenatal and perinatal rats}

\section{Melatonin and FMT improve gut health by increasing intestinal villus height/crypt depth and enhancing antioxidation in antenatal and perinatal rats}

Gut health in antenatal and perinatal animals, which is affected by intestinal oxidative stress and microbiota, is implicated in their nutrition absorption, reproduction and lactation. To investigate the effects of melatonin and melatonin-remodeled FMT on guts, antenatal and perinatal female rats were treated with melatonin and FMT, respectively. The timeline of treatments and sample collection in this study (Fig. 1A). The results showed that the villus height/crypt depth (V/C) of duodenum, jejunum and ileum apparently increased, but colon V/C did not change on antenatal day 5 (A5) and perinatal day 3 (P3) rats (Fig. 1B-F). It was similar to that of the villus height (Supplementary Fig. 1A-D). Additionally, crypt depths of duodenum, jejunum, ileum and colon tended to increase, but not reach statistical significance (Supplementary Fig. 1E-H). Melatonin actions in reducing ulcer formation seemingly to directly scavenge toxic oxygen-based reactants and possibly to promote antioxidative enzyme activities. Here, we found that both melatonin and FMT markedly decreased ROS production of intestinal tissue on A5 and P3 rats (Fig. $1 \mathrm{G}$ and $\mathrm{H}$ ), meanwhile reduced the levels of T-AOC and MDA (Fig. $1 \mathrm{l}$ and $\mathrm{J}$ ). Compared with control group, melatonin upregulated the protein levels of GPx5, SOD1, SOD2 and CAT in intestinal tissues of A5 and P3 rats, and FMT increased the levels of above proteins in gut tissues of P3 rats (Fig. 1K-0). Interestingly, FMT added the protein levels of SOD1 and SOD2 (Fig. 1M and N), but did not change that of GPx5 and CAT in intestinal tissues of A5 rats (Fig. $1 \mathrm{~L}$ and 0 ). Taken together, melatonin and FMT improved gut health by increasing the intestinal V/C and enhancing antioxidation in antenatal and perinatal rats.

\section{Melatonin and FMT inhibit intestinal autophagy and inflammation}

Melatonin and FMT markedly restrained gut autophagy through reducing cellular autophagic vacuoles (Fig. 2A and B), downregulated the protein levels of LC3B-I, LC3B-II, ATG7 and BECN1, whereas upregulated the protein levels of P62 (Fig. 2C-I). Additionally, the intestinal epithelial monolayer plays an 
important role in the development of inflammatory bowel disease, particularly as a target and producer of TNF [27]. Our results indicated that melatonin and FMT significantly inhibited gut inflammatory (Fig. 2J), decreased the contents of TNFa, IL-1 3 , IL-6 and IL-10 (Fig. 2K-N). Likewise, melatonin and FMT upregulated the protein levels of HSP72, but downregulated the protein levels of NF-KB (Fig. 2O-Q). Therefore, melatonin and FMT attenuate autophagy and inflammation to protect gut in antenatal and perinatal rats.

\section{Part 2. Melatonin, FMT and FC ameliorate gut health in early weaned rats}

\section{Melatonin, FMT and FC increase intestinal V/C to ameliorate gut health by enhancing antioxidation in early weaned rats}

Gut health of early weaned animals is beneficial to absorb nutrition for adapting new food condition and decreasing mortality. To detect the effect of melatonin, FMT and FC on intestine of early weaned rats, rats were treated with melatonin, FMT and FC, respectively. In this study, the timeline of treatments and sample collection (Fig. 1A). The results showed that the V/C were increased on the day 3 after weaning (Fig. 3B-F). Likewise, the villus heights of duodenum, jejunum, ileum and colon significantly increased (Supplementary Fig. 2A-D), whereas the crypt depths did not change (Supplementary Fig. 2E-H). Moreover, melatonin, FMT and FC apparently decreased ROS production of intestinal tissues on day 3 after weaning (Fig. $3 \mathrm{G}$ and $\mathrm{H}$ ), meanwhile reduced the contents of TAOC and MDA (Fig. $3 \mathrm{I}$ and $\mathrm{J}$ ), and upregulated the protein levels of GPX5, SOD1, SOD2 and CAT in gut tissues (Fig. 3K-0). Although FC also ameliorated gut antioxidation, its effect was not well as that of melatonin and FMT. Herein, melatonin, FMT and FC increased intestinal V/C to ameliorate gut health by inhibiting oxidative stress in early weaned rats.

\section{Melatonin, FMT and FC suppress autophagy and inflammation and reduce intestinal permeability}

To further reveal the regulatory mechanism of melatonin, FMT and FC in gut health in early weaned rats, we investigated inflammation, autophagy and permeability of gut tissue. The results showed that melatonin, FMT and FC markedly restrained gut autophagy (Fig. 4A and B), downregulated the protein levels of LC3B-I, LC3B-II, ATG7 and BECN1, whereas upregulated the protein levels of P62 (Fig. 4C-I). Furthermore, melatonin, FMT and FC significantly inhibited gut inflammatory (Fig. 4J), decreased the contents of TNFa, IL-1 1 IL-6 and IL-10 (Fig. 4K-N). Meanwhile, melatonin, FMT and FC upregulated the protein levels of HSP72 (Fig. 40 and P), but downregulated the protein levels of NF-KB (Fig. 40 and Q). Interestingly, melatonin, FMT and FC reduced intestinal permeability (Fig. 5A) through increasing tight junction protein (Fig. 5B) and the protein levels of ZO-1, Occludin and Claudin in gut tissue (Fig. 5C-F). In summary, melatonin, FMT and FC attenuated inflammation and autophagy, and reduced intestinal permeability to improve gut health in early weaned rats.

Part 3. Melatonin directly enhances antioxidation and attenuates autophagy and apoptosis in $\mathrm{H}_{2} \mathrm{O}_{2}-$ induced IEC6 
Oxidative stress damages intestinal epithelial cells and causes cell autophagy [28]. IEC6 treated with $\mathrm{H}_{2} \mathrm{O}_{2}$ can well mimic impairment of intestinal epithelial cells. To uncover how melatonin regulates antioxidation and autophagy, we performed the experiments of IEC6 treated with $\mathrm{H}_{2} \mathrm{O}_{2}$ and melatonin. The experiment was performed to reveal the effect of Mel on anti-oxidation, anti-autophagy and antiapoptosis in IEC6 cells (Fig. 6A). The results showed that $\mathrm{H}_{2} \mathrm{O}_{2}$ induced oxidation and autophagy in a dose- and time-dependent manner in IEC6 (Fig. 6B; Supplementary Fig. 3A and B), downregulated the protein expression levels of SOD1, SOD2, GPX5, CAT and P62, but upregulated the protein expression levels of LC3B-I, LC3B-II, BECN1 and ATG7 (Supplementary Fig. 3C-L). Moreover, melatonin apparently suppressed ROS production and strengthened antioxidation in $\mathrm{H}_{2} \mathrm{O}_{2}$-induced IEC6 (Fig. 6B), meanwhile restored the protein expression levels of P62, SOD1, SOD2, GPX5 and CAT (Fig. 6C-G). Interestingly, melatonin inhibited autophagy and rescued cell viability in $\mathrm{H}_{2} \mathrm{O}_{2}$-induced IEC6 (Fig. 6H-J). Meanwhile, melatonin decreased the LC3B-II/ LC3B-I and upregulated the protein expression level of P62 in $\mathrm{H}_{2} \mathrm{O}_{2}-$ induced IEC6 (Fig. 6K-M). Based on the results, melatonin attenuated autophagy caused by $\mathrm{H}_{2} \mathrm{O}_{2}$ through a reduction in an excessive oxidative stress.

Apoptosis of intestinal epithelial cells following oxidative stress is involved in the pathogenesis of various gastrointestinal diseases [29]. We therefore investigated the mechanism on melatonin modulating apoptosis of IEC6. The results indicated that $\mathrm{H}_{2} \mathrm{O}_{2}$ distinctly caused apoptosis, but melatonin inhibited apoptosis induced by $\mathrm{H}_{2} \mathrm{O}_{2}$ in IEC6 (Fig. $6 \mathrm{~N}$ ), rescued mitochondria dysfunction through reducing the number of apoptotic body (Fig. 60). Moreover, melatonin partially restored the protein levels of Cleaved-caspase-3, Bcl-2, Bcl-XL, full length/cleaved PARP (Fig. 6P-T), and inhibited upregulation of the protein levels of both mitochondria-Cytochrome $\mathrm{C}-1$ and cytoplasm-Cytochrome $\mathrm{C}-1$ by $\mathrm{H}_{2} \mathrm{O}_{2}$ in IEC6 (Fig. $6 \mathrm{U}, \mathrm{V})$. Taken together, melatonin inhibited apoptosis of intestinal epithelial cells following oxidative stress, possibly causing a decrease of intestinal villus height.

\section{Part 4. Melatonin, FMT and FC improve gut health by reprogramming gut microbiota}

\section{Melatonin, FMT and FC remodel gut microbiota}

Gut microbiota is highly associated with intestinal health, so we further detected fecal microbiota compositions by sequencing the fecal bacterial 16S rRNA in A5 and P3 rats, and rats on the day 3 after weaning. Experiment design for feces bacterial 16S rDNA gene amplicon sequencing analysis was as shown in Fig. 7A. In this study, total tags of 64734 raw reads were generated from each sample (Supplementary Fig. S4A). After removing the low-quality sequences, more than 50000 clean tags were clustered into OTUs (Supplementary Fig. S4B and C). Antenatal and perinatal rats, as well as early weaned rats treated with melatonin, FMT and FC exhibited a greater diversity of microbiota evidenced by the increased Shannon and Simpson indexes compared to controls (Fig. 7B and C). We analyzed overall differences in $\beta$-diversity using PCoA plot, weighted and unweighted UniFrac distance. Controls and treatments showed a distinct clustering of microbial community, structure, while the FMT and FC groups had a similar structure to that of the Mel groups (Fig. 7D and E; Supplementary Fig. S4D-G). The overall 
microbial composition in 10 groups differed at the phylum, order and genus species levels (Fig. 7F and $\mathrm{G})$. At the genus level, compared with control groups, the relative abundances of Allobaculum and Faecalibaculum were increased in the treatment groups, and the relative abundances of Bifidobacterium were augmented in AMel, AFMT, WMel WFMT and WFC groups (Fig. 7H). Based on the analysis presented above, the abundance changes of these intestinal microbiota which produced SCFAs may be involved in gut health.

\section{Melatonin, FMT and FC improve gut health by increasing gut microbiota produced SCFAs}

SCFAs including acetic, propionic and butyric acids are the main metabolic products of anaerobic bacteria fermentation in the intestine [30]. These fatty acids have been recognized as potential mediators involved in the effects of gut microbiota on intestinal antioxidation, autophagy and immune function. Hence the fecal SCFAs concentrates were detected in this study. The results indicated that both Mel and FMT increased the concentrations of total SCFAs, acetic and butyric acid in 5P and 3A rats (Fig. 8A, B, D), whereas did not change the concentrations of propionic acid (Fig. $8 \mathrm{C}$ ). For early weaned rats, the results were in accord with those of $5 \mathrm{P}$ and $3 \mathrm{~A}$ rats $(\mathrm{Fig} .8 \mathrm{~F}-\mathrm{H})$. On the basis of the data, SCFAs, especially acetic and butyric acids produced by remodeling gut microbiota, might serve as a potential effect of melatoninmediated gut health improvement. Here, we cannot exclude that the other factors caused by altered microbiota affect gut health, such as some antioxidants, toxins and other metabolites.

Furthermore, the abundance change of Lactoaccillus was not affected fecal acetic acid concentration (Fig. 8I), but those of Allobaculum, Bifidobacterium and Faecalibaculum were related to fecal acetic acid concentration (Fig. 8J-L). The abundance changes of Lactoaccillus and Allobaculum were not changed fecal butyric acid concentrations (Fig. 8M), whereas those of Bifidobacterium and Faecalibaculum were related to fecal butyric acid concentrations (Fig. 8N-P). These results showed that Allobaculum, Bifidobacterium and Faecalibaculum contributed to acetic and butyric acid production to protect gut health. Overall, our results suggested that melatonin, FMT and FC increased the gut microbiota strains which produced more SCFAs to ameliorate gut health through increasing intestinal V/C and inhibiting oxidative stress, autophagy, inflammatory and apoptosis (Fig. 9).

\section{Discussion}

Gut health is vital important for mammal to not only improve nutrient absorption, but also resist pathogen, toxin and allergic compound. For antenatal, perinatal and early weaned animals, intestinal health is also implicated in reproduction, lactation and mortality rate after weaning. Melatonin is a high effective antioxidant which protects essential cell elements such as nucleus, mitochondria, and endoplasmic reticulum [31-33]. Additionally, it is now known to be present in the gastrointestinal tract, and its functions in the gut generally seem to be protective of the mucosa from erosion and ulcer formation [34]. In the present study, melatonin directly ameliorated intestinal tissue antioxidation, inhibited inflammation and autophagy in antenatal, perinatal and early weaned rats. Likewise, in vitro experiments further revealed that melatonin enhanced antioxidation to attenuate autophagy and 
apoptosis in $\mathrm{H}_{2} \mathrm{O}_{2}$-induced IEC6. Therefore, melatonin increased intestinal V/C to improve gut health by suppressing oxidative stress, inflammation, autophagy and apoptosis via downregulating the levels of ROS and inflammatory factor, reducing autophagic vacuole and apoptotic body. Interestingly, melatonin not only increases autophagy, but also blocks autophagy [35, 36]. In this study, melatonin blocked autophagy through decreasing autophagic vacuole to exert its gut-protective role against intestinal oxidative damage. On the basis of data discussed herein, our results confirm and extend previous findings that intestinal melatonin is highly effective protector against oxidative stress-induced lesions [37, 38].

Next, we investigated how melatonin-reprogrammed FMT function on the gut in antenatal and perinatal rats. FMT is the infusion of liquid filtrate feces from a healthy donor into the gut of a recipient to cure a specific disease, including Parkinson's disease, fibromyalgia, chronic fatigue syndrome, multiple sclerosis, obesity and insulin resistance $[39,40]$. In fact, FMT is the transfer of minimally manipulated, prescreened donor stool into the gastro-intestinal tract of a patient, with the aim of ameliorating the dysbiotic state by increasing overall diversity and restoring the functionality of the microbiota [41]. Increasing literatures showed that gut microbiota was sensitive to melatonin exposure and melatonin treatment markedly shapes or reverses gut microbiota in healthy or sick animal models [42-45]. The variation of specific bacteria in melatonin-reprogrammed gut microbiota effect obesity and insulin resistance [46, 47]. Decrease of Firmicutes-to-Bacteroidetes ratio and increase of abundance of mucin-degrading bacteria Akkermansia were associated with healthy mucosa [14]. In this study, the composition and abundance of gut microbiota was different among AMel, AFMT, PMel and PFMT groups, and more Allobaculum, Bifidobacterium and Faecalibaculum were implicated in gut health because they produced acetic and butyric acid. Here, we need point out that the gastrointestinal tract responds with increment of melatonin to feeding, but pineal gland production of melatonin remains unaffected by feeding [48]. Moreover, pineal gland and small bowel are highly effective in the synthesis and secretion of melatonin [49], but it need be further explored whether exogenous melatonin affects synthesis of endogenous melatonin. Overall, we suggested that melatonin targeted reconstitution of the gut microbiota against intestinal oxidative stress.

Although the majority of the results are consistent between studies, some potential taxa and metabolites may appear discrepant and even contrasting, thereby supporting the need for appropriate studies to ascertain the role of one or several specific bacteria as potential beneficial bacteria to provide protection against specific intestinal diseases. Developments in high-throughput microbial genomic sequencing have given novel insight into the potential contribution of the gut microbiota to health. Moreover, restoration of the gut microbiota to a premorbid state is a key novel therapeutic approach, and FMT is gaining increasing importance in both the clinical and research settings. To date, although a large number of trials are ongoing worldwide exploring FMT potential therapeutic indications, it is only recommended in the treatment of recurrent Clostridioides difficile infection [50]. On the basement of our findings, we might someday be able to mine for melatonin-reprogrammed gut bacterial strains that can be used in the diagnosis or treatment of intestinal diseases, such as Allobaculum, Bifidobacterium and Faecalibaculum in this study. 
In general, mammalian weaning resulted in reduction of intestinal $\mathrm{V} / \mathrm{C}$ and damaged intestinal integrity [51]. In this study, our findings revealed that melatonin and melatonin-remodeled FMT could increase intestinal V/C, ameliorate gut antioxidation, inhibit autophagy and inflammation, and decrease intestinal permeability in early weaned rats. Interestingly, FC also functioned above effects, but why? Animals subjected to oxidative stress exhibited significant loss of crypt architecture, severe villous epithelial atrophy and degeneration, and shortening of villi [52-54]. Here, melatonin and FMT were beneficial to gut health through inhibiting oxidative stress in early weaned rats. Therefore, we thought that the early weaned rats in FC group could get beneficial microbiota via eating or licking the dung of their mothers treated with melatonin to improve gut health. Furthermore, abundance of Allobaculum, Bifidobacterium and Faecalibaculum markedly increased in FC group, hinting that these important changes which caused differences in intestinal microbial products including total SCFAs, acetic and butyric acid in early weaned rats. Interestingly, Faecalibaculum produced butyric acid which protect gut health [55-57]. In addition, Bifidobacterium also produced moderate $\mathrm{H}_{2} \mathrm{O}_{2}$ to activates the body's production of catalase which kills gram negative bacteria such as Shigella and Salmonella [58]. Although we still do not exclude the possibility that altered microbiota did not produce these bacteriocins or changed them might be caused by other factors, metabolic SCFAs from remodeled intestinal microbiota ameliorated gut antioxidation, inhibited autophagy and inflammation in early weaned rats in FC group. However, more experimental studies need to be performed to further uncover the composition and function of the reprogrammed microbiota and their bacteriocins in the future. In the current study, our findings suggested that FC improved gut health of the foster rats through remodeled microbiota, especially more Allobaculum, Bifidobacterium and Faecalibaculum. Additionally, lower levels of melatonin and SCFA concentration correlated with increased intestinal permeability $[59,60]$. In accord with these studies, intestinal permeability decreased by adding exogenous melatonin or increasing levels of SCFAs. Here, we need point out that early weaned rats ate or licked the dung from female rats treated with melatonin, in which might contain undetected infinitesimal melatonin. Overall, although the regulation and effect the gut microbiota were affected by many factors and were very complex, the potential for FC manipulation to ameliorate gut health is a novel area of rapid growth and great promise.

In conclusion, melatonin, FMT and FC increased gut microbiota which produced SCFAs to improve gut health through enhancing antioxidation, inhibiting autophagy, inflammatory and apoptosis. We suggest that precise manipulating intestinal microbiota via melatonin, its-reprogrammed FTM and FC will become promising strategies for therapy of intestinal disorders caused by oxidative stress in antenatal, perinatal and weaned mammals.

\section{Materials And Methods}

\section{Animals}

The male Sprague-Dawley rats (10-12 weeks) were purchased from The Fourth Military Medical University (Xian, Shaanxi, China). The rats were housed in a pathogen-free rat colony (temperature, $25 \pm$ $2^{\circ} \mathrm{C}$; relative humidity, $45 \%-60 \%$; lighting cycle, $12 \mathrm{~h} /$ day, 08:00-20:00 for light) and had free access to 
food and drinking water. The normal chow diet was purchased from Beijing HFK Bioscience Co. Ltd. (Beijing, China). After superovulation of rats by an intraperitoneal injection of 10 IU PMSG (Sigma, St Louis, MO, USA), the female rats were mated. The pregnant rats were selected for experiments. The animal experiments were all approved by the Research Ethics Committee of Northwest A\&F University (2011-31101684).

\section{Experiment design}

For the pregnant and lactation rat experiments (Fig. 1A), a total of 30 pregnant rats were randomly divide into 3 groups, including control, melatonin (Mel) and FMT group. Control and FMT groups: rats received a basal diet and normal drinking water; Mel group: a basal diet and drinking water containing melatonin ( $0.2 \mathrm{~g} / \mathrm{L} ; \mathrm{M} 5250$, Sigma, MO, USA). After 3 days of melatonin supplementation, the fecal contents of rats were collected and diluted with phosphate buffer saline (PBS) through modified procedure according to precious method $[3,23,24]$. Briefly, fresh fecal samples were collected from Mel group in sterile PBS (100 $\mathrm{mg} / \mathrm{mL}$ ). The suspension was homogenized by vortex for $1 \mathrm{~min}$ and centrifuged at $1000 \mathrm{~g}$ for $3 \mathrm{~min}$. The resulting supernatant was collected into new sterile tubes and used for oral gavage. The fecal contents dilution $(0.1 \mathrm{~mL} / \mathrm{rat})$ was feed into rats in FMT group each day until the day 5 before delivery and the day 3 after delivery, respectively. Five rats were sacrificed to collect duodenum, jejunum, ileum and colon in each group. These intestinal tissues were fixed in $2.5 \%$ glutaraldehyde solution and $2.5 \%$ paraformaldehyde solution and saved at $-80^{\circ} \mathrm{C}$. The 30 fresh fecal samples on the day 5 before delivery and the day 3 after delivery were used for bacterial 16S rDNA gene amplicon sequencing analysis were stored at $-80^{\circ} \mathrm{C}$ before genomic DNA extraction.

For the weaned rat experiments (Fig. 3A), a total of 80 suckling rats at 11 days of age (weaned at 21 days of age) were randomly selected for four groups including control, Mel, FMT and FC group. Control and FMT groups: suckling rats fed a basal diet and normal drinking water; Mel group: suckling rats fed a basal diet and drinking water containing melatonin $(0.2 \mathrm{~g} / \mathrm{L}) ; \mathrm{FC}$ group: lactating rats received a basal diet and drinking water containing melatonin $(0.2 \mathrm{~g} / \mathrm{L})$. After 3 days of melatonin supplementation, the fecal contents dilution $(0.1 \mathrm{~mL} / \mathrm{rat})$ was feed into suckling rats in FMT group each day until 24 days of age. Similarly, five rats were sacrificed to collect serum, duodenum, jejunum, ileum and colon in each group. The 20 fresh fecal samples on the day 3 after weaning were used for bacterial sequencing analysis.

\section{Hematoxylin and eosin staining}

Hematoxylin and eosin (H\&E) staining was performed as previously described [25]. Briefly, the rat intestinal tissue, including duodenum, jejunum, ileum and colon, were fixed with $4 \%$ paraformaldehydePBS overnight, and then dehydrated and embedded in paraffin blocks. The $5 \mu \mathrm{m}$ sections were deparaffinized, hydrated and stained with H\&E. Intestinal villus height and crypt depth were measured using ImageJ software.

\section{Intestinal antioxidant capacity}

Page $11 / 30$ 
The levels of malondialdehyde (MDA) and total antioxidant capacity (TAOC) in intestinal tissues were measured by spectrophotometric methods according to manufacturer instructions of assay kits (Nanjing Jiancheng, Nanjing, China). All samples were measured by a UV/visible spectrophotometer (UV-2450, Shimadzu, Kyoto, Japan).

\section{ROS generation assay}

The fresh intestinal tissues were collected and frozen at $-80^{\circ} \mathrm{C}$. The samples were sliced into $5-10 \mu \mathrm{m}$ pieces and stained with DHE at $37^{\circ} \mathrm{C}$ for $30 \mathrm{~min}$. The sections were washed on time with PBS for 10 minutes and stained with DAPI. Likewise, intestinal epithelial cells were washed 3 times with PBS and incubated with $10 \mu \mathrm{M}$ DCFH-DA (Invitrogen, Eugene, OR, USA) for $30 \mathrm{~min}$, and then washed with PBS and stained with DAPI. The sections and cells were captured with a Zeiss 710 laser scanning confocal microscope. The ROS assay kit (Beyotime Institute of Biotechnology, Shanghai, China) were used to measure ROS concentration according to the manufacturer's instructions. Briefly, the crushed intestinal tissues were incubated with $10 \mu \mathrm{M}$ DCFH-DA for $30 \mathrm{~min}$, and then were measured by UV/visible spectrophotometer (UV-2450, Shimadzu, Kyoto, Japan).

\section{Transmission electron microscopy}

The fresh ileum tissues or the rat intestinal epithelial cell line IEC6 cells were fixed in $2.5 \%$ glutaraldehyde solution at $4^{\circ} \mathrm{C}$ overnight and incubated with PBS containing $2 \%$ OsO4 for $4 \mathrm{~h}$. The ileum tissues then were dehydrated in a graded series of ethanol $(30 \%, 50 \%, 70 \%, 80 \%, 90 \%, 95 \%$ and $100 \%)$ for 10 min at each step for 2 times and then transferred into mixture of alcohol and spurr resin (v:v = 1:1, v:v $=1: 2, v: v=$ $1: 3)$ for $12 \mathrm{~h}$ and spurr resin for $24 \mathrm{~h}$ for 2 times. Ultrathin sections were obtained using a diamond knife and stained with uranyl acetate, and then specimens were observed by TEM (JEM-1011, JEOL, Japan).

\section{Immunohistochemistry}

Immunohistochemistry stains were detected using IHC kit (MaiXin, China). Briefly, the tissues were cut into 3-10 $\mu \mathrm{m}$ long pieces and blocked with $1 \% \mathrm{w} / \mathrm{v}$ BSA for $1 \mathrm{~h}$, and then anti-NF-KB antibody were incubated overnight at $4^{\circ} \mathrm{C}$. Next, the samples were dehydrated in an ethanol (30-100\%) gradient and treated with xylene to increase the transparency of slides.

\section{ELISA assay}

The levels of IL-6, IL-1 $\beta$, IL-10 and TNF-a were determined using ELISA kits (Raybiotech, GA, USA) according to the manufacturer's instructions.

\section{Intestinal permeability analysis}

Intestinal permeability was assessed using the FITC-dextran (4-kDa) permeability assay. On 24 days of age, the weaned rats were starved for $12 \mathrm{~h}$, then feed with FITC-dextran $(4.4 \mathrm{mg} / \mathrm{kg} \mathrm{BW})$ by oral gavage. 
After $5 \mathrm{~h}$, whole blood was collected and serum was isolated. Serum was diluted with equal volume of PBS and tested in duplicate. The pictures of FITC-dextran were captured with fluorometer.

\section{Western blotting analysis}

Total protein was isolated from intestinal tissues and cells by incubating in RIPA buffer containing protease inhibitor cocktail for $30 \mathrm{~min}$ and then centrifuged at $12,000 \times \mathrm{g}$ for $10 \mathrm{~min}$ at $4^{\circ} \mathrm{C}$ to remove the precipitate. The total protein content was determined using the BCA Protein Assay Kit (Pierce), and $30 \mu \mathrm{g}$ proteins were separated by a reducing SDS-PAGE electrophoresis on $10 \%$ or $15 \%$ Bis-Tris gels, transblotted onto nitrocellulose membranes and probed with different primary antibodies: GPx5 (18731-1AP, 1 : 1000, Proteintech), SOD1 (10269-1-AP, $1: 1000$, Proteintech), SOD2 (13194S, $1: 1000$, CST), CAT (14097S, 1 : 1000, CST), HSP72, NF-KB (8242S, 1 : 1000, CST), LC3B (3868S, $1: 1000$, CST), P62 (23214S, 1 : 1000, CST), ATG7 (2631S, 1 : 1000, CST), Beclin1 (11306-1-AP, 1 : 1000, Proteintech), ZO1 (ab221547, 1 : 1000, Abcam), Occludin (27260-1-AP, 1 : 1000, Proteintech), Claudin-1 (13050-1-AP, 1 : 1000, Proteintech), Bcl-2 (ab194583, 1 : 1000, Abcam), Bcl-XL (26967-1-AP, $1: 500$, Proteintech), Cleaved capsase-3 (ab49822, 1 : 1000, Abcam), PARP (13371-1-AP, 1 : 1000, Proteintech), Cytochrome C (10993-1AP, 1 : 1000, Proteintech), $\beta$-actin (4967S, $1: 1000$, CST) and COX IV (ab202554, $1: 500$, Abcam). The gray values of the bands were measured by ImageJ software and then normalized to $\beta$-actin or COX IV content.

\section{Cell culture and treatment}

The rat intestinal epithelial cell line IEC- 6 were purchased from the American Type Culture Collection (ATCC, USA). The cells were cultured in DMEM-F12 medium supplemented with 10\% FBS (Life Technologies) in a humidified $5 \% \mathrm{CO}_{2}$ incubator at $37^{\circ} \mathrm{C}$. For $\mathrm{H}_{2} \mathrm{O}_{2}$ and melatonin treatments, cells were pretreated with melatonin $(10 \mu \mathrm{M})$ for $24 \mathrm{~h}$, followed by $\mathrm{H}_{2} \mathrm{O}_{2}(200 \mu \mathrm{M})$ stimulation for $2 \mathrm{~h}$ before they were used for the next assay.

\section{Cell viability assay}

IEC-6 viability was measured using Cell Counting Kit-8 (Beyotime Institute of Biotechnology, Shanghai, China) according to the manufacturer's instructions.

\section{Evaluation of autophagosome}

IEC-6 were seeded in 24-well plates with $1 \mathrm{~cm}$ coverslips, and the cells grown to $80 \%$ confluence prior to transient transfection with the GFP-MAP1LC3B (Beyotime Institute of Biotechnology, Shanghai, China). After $24 \mathrm{~h}$ cultured, the cells were treated with $10 \mu \mathrm{M}$ melatonin and $200 \mu \mathrm{M} \mathrm{H}_{2} \mathrm{O}_{2}$. Finally, the cells were washed with PBS for 3 times, and observed under a laser-scanning confocal microscope (Zeiss LSM 710 META, Oberkochen, Germany).

\section{Immunofluorescence}


For immunofluorescence analysis, IEC6 were fixed in 4\% paraformaldehyde for $10 \mathrm{~min}$ and were permeabilized in $0.5 \%$ Triton $\mathrm{X}-100$ for $20 \mathrm{~min}$ at room temperature and blocked in 1\% BSA for $30 \mathrm{~min}$. Next, the cells were incubated with primary antibodies against Cleaved caspase-3 overnight at $4^{\circ} \mathrm{C}$. After that, the samples were washed three times with PBS for 10 min each time and incubated with secondary antibodies for $2 \mathrm{~h}$. The nuclei were stained with DAPI for $10 \mathrm{~min}$ and then washed one time with PBS for 15 min. Immunofluorescent images were captured with a Zeiss 710 laser scanning confocal microscope.

\section{Gut microbiota analysis}

Total genomic bacterial DNA of fecal mixture of the duodenum, jejunum, ileum and colon was extracted using an E.Z.N.A. ${ }^{\circledR}$ Stool DNA Kit (Omega Bio-Tek, Norcross, GA, USA) following the manufacturer's instructions. PCR amplification of bacteria DNA in the present study was performed. Briefly, the V3-V4 regions of the bacteria 16S ribosomal RNA gene were amplified by PCR as following: $95^{\circ} \mathrm{C}$ for $2 \mathrm{~min}$, followed by 25 cycles at $95^{\circ} \mathrm{C}$ for $30 \mathrm{~s}, 55^{\circ} \mathrm{C}$ for $30 \mathrm{~s}$, and $72^{\circ} \mathrm{C}$ for $30 \mathrm{~s}$, and a final extension at $72^{\circ} \mathrm{C}$ for 5 min. The primers used are 338F 5'-ACTCCTACGGGAGGCAGCA-3' and 806R 5'-

GGACTACHVGGGTWTCTAAT-3'. PCRs were performed in triplicate $20 \mu \mathrm{L}$ mixture containing $4 \mu \mathrm{L}$ of $5 \times$ FastPfu Buffer, $2 \mu \mathrm{L}$ of $2.5 \mathrm{mM}$ dNTPs, $0.8 \mu \mathrm{L}$ of each primer $5 \mu \mathrm{M}, 0.4 \mu \mathrm{L}$ of FastPfu DNA polymerase, and $10 \mathrm{ng}$ of template DNA.

Amplicons were extracted from $2 \%$ agarose gel, purified by the AxyPrep DNA gel extraction kit (Axygen Biosciences, Union City, CA, USA), and quantified by QuantiFluorTM-ST (Promega, Madison, WI, USA) according to the manufacturer's protocols. Then purified amplicons were pooled in equimolar amounts and paired-end sequenced on an Illumina MiSeq platform according to the standard protocols by a commercial company (Novogene, Beijing, China). The raw reads were deposited into the NCBI Sequence Read Archive (SRA) database (Accession Number: SUB2623584).

Raw data were firstly conjuncted by using the FLASH analysis tool (V1.2.7, http://ccb.jhu.edu/software/FLASH/), and then filtered with the Quality Control software package (V1.7.0, http://qiime.org/scripts/split_libraries_fastq.html). To obtain the effective tags, the UCHIME algorithm (http://www.drive5.com/usearch/manual/uchime_algo.html) was used to remove the chimera sequences. The reads were picked to form distinct OTUs using Uparse software (http://drive5.com/uparse/) at $97 \%$ of sequence similarity, and then were classified to different levels by comparing to GreenGenes database using PyNAST software (V1.2).

\section{SCFAs}

For detection of SCFAs, fecal samples were measured using the previous method [22,26]. Briefly, the fecal mixture samples were treated as follows: $0.3 \mathrm{~g}$ of feces was added to $1.2 \mathrm{~mL}$ sterile saline, evenly dispersed by stirring with a glass rod, and left to stand for $5 \mathrm{~min} ; 600 \mu \mathrm{L} 50 \%$ sulfuric acid (Sigma Aldrich) and $1.2 \mathrm{~mL}$ ether were added, standing for $10 \mathrm{~min}$ with intermittent shaking. The supernatant was obtained by centrifugation at $5000 \mathrm{rpm}$ for $30 \mathrm{~min}$ and used for chromatographic analysis. The content of SCFA were measured by GC-MS (Agilent, USA). 


\section{Statistical analysis}

Statistical analyses were performed using the SPSS 23.0 statistical software package (SPSS Inc, Chicago, IL, USA). The variables were first tested for normality (Shapiro-Wilk test) and homoscedasticity (Levene's test) and followed with Student's $t$ test. The data were compared by analysis of variance (ANOVA) and differences located with Duncan's multiple range test. Correlation analysis between microbiota and SCFAs was conducted by Pearson correlation analysis. All data of each group were presented as mean \pm SEM and statistical significance was set at $P<0.05$.

\section{Abbreviations}

Mel: Melatonin; FMT: Fecal microbiota transplantation; FC: Foster care; V/C: Intestinal villus height/crypt depth; SCFAs: Short-chain fatty acids; MDA: Malondialdehyde; TAOC: Total antioxidant capacity; TEM: Transmission electron microscopy; H\&E: Hematoxylin and eosin; PBS: phosphate buffer saline; GPx5: Glutathione peroxidase-5; SOD1: Superoxide dismutase 1; SOD2: Superoxide dismutase 5; CAT: Chloramphenicol acetyltransferase; A5: Antenatal day 5; P3: Perinatal day 3; TNFa: Tumour necrosis factor alpha; IL-1ß: Interleukin-1beta; IL-6: Interleukin-6; IL-10: Interleukin-10; HSP72: Heat shock protein 72; NF-KB: Nuclear factor-kappaB; LC3B-I: Light chain 3B-I; LC3B-II: Light chain 3B-II; ATG7: Autophagyrelated gene 7; BECN1: Beclin1; P62: Sequestosome 1; ZO-1: Zonula occludens-1; IEC6: Rat intestinal epithelial cells 6; Bcl-2: B cell lymphoma-2; Bcl-xL: B-cell lymphoma-extra large; PARP: Poly(ADP-ribose) polymerase.

\section{Declarations}

\section{Acknowledgements}

Not applicable.

\section{Authors' contributions}

WP provided financial support and conceived the study. YW, WS, RC and XY performed most of the experiments, wrote the manuscript. WP, JW, GC and LG revised the manuscript. JW, GC, LG, WY, CW and ZK conducted the animal experimentation, histological analysis and biochemical analysis. All authors read and approved the final manuscript.

\section{Funding}

This work was supported by the National Natural Science Foundation of China (No. 31872979) and China Agriculture Research System of MOF and MARA (CARS-35).

\section{Availability of data and materials}


The 16S rRNA gene sequencing data of the discovery cohort were submitted to the SRA database in NCBI with the accession number PRJNA778474 on antenatal and perinatal rats and PRJNA778499 on weaned rats, respectively. Moreover, the data supporting the findings of this study are available from the corresponding author upon request.

\section{Ethics approval and consent to participate}

This study does not involve the use of human subjects. The ethical statements regarding animal experimentation are included in the "Materials and methods" section.

\section{Consent for publication}

Not applicable.

\section{Competing interests}

All authors have no competing interests.

\section{Author details}

Key Laboratory of Animal Genetics, Breeding and Reproduction of Shaanxi Province, College of Animal Science and Technology, Northwest A\&F University, Yangling, Shaanxi, 712100, China.

\section{References}

1. 1. P.D. Cani, Human gut microbiome: hopes, threats and promises, Gut 67 (2018) 1716-1725. https://doi: 10.1136/gutjnl-2018-316723.

2. 2. T. Hrncir, L. Hrncirova, M. Kverka, H. Tlaskalova-Hogenova, The role of gut microbiota in intestinal and liver diseases, Lab. Anim. 53 (3) (2019) 271-280. https://doi: 10.1177/0023677218818605.

3. 3. J. Hu, L. Ma, Y. Nie, J. Chen, W. Zheng, X. Wang, et al., A microbiota-derived bacteriocin targets the host to confer diarrhea resistance in early-weaned piglets, Cell Host Microbe 24 (6) (2018) 817-832. https://doi: 10.1016/j.chom.2018.11.006.

4. 4. L.P. Smits, K.E. Bouter, W.M. de Vos, T.J. Borody, M. Nieuwdorp, Therapeutic potential of fecal microbiota transplantation, Gastroenterology 145 (2013) 946-953. https://doi:

10.1053/j.gastro.2013.08.058.

5. 5. S. Lukovac, C. Belzer, L. Pellis, B.J. Keijser, W.M. de Vos, R.C. Montijn, et al., Differential modulation by Akkermansia muciniphila and Faecalibacterium prausnitzii of host peripheral lipid metabolism and histone acetylation in mouse gut organoids, mBio. 5 (4) (2014) e01438-14. https://doi: 10.1128/mBio.01438-14.

6. 6. W. Jia, G. Xie, W. Jia, Bile acid-microbiota crosstalk in gastrointestinal inflammation and carcinogenesis, Nat. Rev. Gastroenterol. Hepatol. 15 (2) (2018) 111-128. https://doi: 10.1038/nrgastro.2017.119. 
7. 7. Wlodarska M, Luo C, Kolde R, d'Hennezel E, Annand JW, Heim CE, et al., Indoleacrylic Acid Produced by Commensal Peptostreptococcus Species Suppresses Inflammation, Cell Host Microbe 22 (1) (2017) 25-37. https://doi: 10.1016/j.chom.2017.06.007.

8. 8. T.R. Whitehead, N.P. Price, H.L. Drake, M.A. Cotta, Catabolic pathway for the production of skatole and indoleacetic acid by the acetogen Clostridium drakei, Clostridium scatologenes, and swine manure, Appl. Environ. Microbiol. 74 (6) (2008) 1950-1953. https://doi: 10.1128/AEM.02458-07.

9. 9. B.B. Williams, A.H. Van Benschoten, P. Cimermancic, M.S. Donia, M. Zimmermann, M. Taketani, et al., Discovery and characterization of gut microbiota decarboxylases that can produce the neurotransmitter tryptamine, Cell Host Microbe 16 (4) (2014) 495-503. https://doi: 10.1016/j.chom.2014.09.001.

10. 10. P.J. Turnbaugh, R.E. Ley, M.A. Mahowald, C.M. Fraser-Liggett, R. Knight, J.I. Gordon, An obesityassociated gut microbiome with increased capacity for energy harvest, Nature 444 (7122) (2006) 1027-1031. https://doi: 10.1038/nature05414.

11. 11. F. Söderquist, I. Sundberg, M. Ramklint, R. Widerström, P.M. Hellström, J.L. Cunningham, The relationship between daytime salivary melatonin and gastrointestinal symptoms in young adults seeking psychiatric care, Psychosom. Med. 81 (1) (2019) 51-56. https://doi: 10.1097/PSY.0000000000000644.

12. 12. R.K. Wong, C. Yang, G.H. Song, J. Wong, K.Y. Ho, Melatonin regulation as a possible mechanism for probiotic (VSL\#3) in irritable bowel syndrome: a randomized double-blinded placebo study, Dig. Dis. Sci. 60 (1) (2015)186-194. https://doi: 10.1007/s10620-014-3299-8.

13. 13. M.C. Dao, A. Everard, J. Aron-Wisnewsky, N. Sokolovska, E. Prifti, E.O. Verger, et al., Akkermansia muciniphila and improved metabolic health during a dietary intervention in obesity: relationship with gut microbiome richness and ecology, Gut 65 (3) (2016) 426-436. https://doi: 10.1136/gutjnl-2014308778.

14. 14. G. Mannino, F. Caradonna, I. Cruciata, A. Lauria, A. Perrone, C. Gentile, Melatonin reduces inflammatory response in human intestinal epithelial cells stimulated by interleukin-1 $\beta$, J. Pineal Res. 67 (3) (2019) e12598. https://doi: 10.1111/jpi.12598.

15. 15. Gareau MG, Sherman PM, Walker WA. Probiotics and the gut microbiota in intestinal health and disease. Nat. Rev. Gastroenterol. Hepatol. 7 (9) (2010) 503-514. https://doi: 10.1038/nrgastro.2010.117.

16. 16. S.J. Konturek, P.C. Konturek, I. Brzozowska, M. Pawlik, Z. Sliwowski, M. Cześnikiewicz-Guzik, et al., Localization and biological activities of melatonin in intact and diseased gastrointestinal tract (GIT), J. Physiol. Pharmacol. 58 (3) (2007) 381-405. http://www.jpp.krakow.pl/journal/archive/09_07/articles/01_article.html

17. 17. L. Pham, L. Baiocchi, L. Kennedy, K. Sato, V. Meadows, F. Meng, et al., The interplay between mast cells, pineal gland and circadian rhythm: Links between histamine, melatonin and inflammatory mediators, J. Pineal Res. 70(2) (2021) e12699. https://doi: 10.1111/jpi.12699. 
18. 18. B. de Luxan-Delgado, B. Caballero, Y. Potes, A. Rubio-González, I. Rodríguez, J. GutiérrezRodríguez, et al., Melatonin administration decreases adipogenesis in the liver of ob/ob mice through autophagy modulation, J. Pineal Res. 56 (2) (2014) 126-133. https://doi: 10.1111/jpi.12104.

19. 19. S. Carloni, G. Riparini, G. Buonocore, W. Balduini, Rapid modulation of the silent information regulator 1 by melatonin after hypoxia-ischemia in the neonatal rat brain, J. Pineal Res. 63 (3) (2017) e12434. https://doi: 10.1111/jpi.12434.

20. 20. B. San-Miguel, I. Crespo, D. Vallejo, M. Álvarez, J. Prieto, J. González-Gallego, et al., Melatonin modulates the autophagic response in acute liver failure induced by the rabbit hemorrhagic disease virus, J. Pineal Res. 56 (3) (2014) 313-321. https://doi: 10.1111/jpi.12124.

21. 21. P. Xu, J. Wang, F. Hong, S. Wang, X. Jin, T. Xue, et al., Melatonin prevents obesity through modulation of gut microbiota in mice, J. Pineal Res. 62 (4) (2017) e12399. https://doi: 10.1111/jpi.12399.

22. 22. J. Yin, Y. Li, H. Han, S. Chen, J. Gao, G. Liu, et al., Melatonin reprogramming of gut microbiota improves lipid dysmetabolism in high-fat diet-fed mice, J. Pineal Res. 65 (4) (2018) e12524. https://doi: 10.1111/jpi.12524.

23. 23. M.J. Hamilton, A.R. Weingarden, M.J. Sadowsky, A. Khoruts, Standardized frozen preparation for transplantation of fecal microbiota for recurrent Clostridium difficile infection, Am. J. Gastroenterol. 107 (5) (2012) 761-767. https://doi: 10.1038/ajg.2011.482.

24. 24. Toral M, Robles-Vera I, de la Visitación N, Romero M, Sánchez M, Gómez-Guzmán M, et al. Role of the immune system in vascular function and blood pressure control induced by faecal microbiota transplantation in rats, Acta. Physiol. 227 (1) (2019) e13285. https://doi: 10.1111/apha.13285.

25. 25. H. Wu, L. Ye, X. Lu, S. Xie, Q. Yang, Q. Yu, Lactobacillus acidophilus alleviated salmonella-induced goblet cells loss and colitis by Notch pathway, Mol. Nutr. Food Res. 62 (22) (2018) 1800552. https://doi: 10.1002/mnfr.201800552.

26. 26. Zhao G, M. Nyman, J.A. Jönsson, Rapid determination of short-chain fatty acids in colonic contents and faeces of humans and rats by acidified water-extraction and direct-injection gas chromatography, Biomed. Chromatogr. 20 (8) (2006) 674-682. https://doi: 10.1002/bmc.580.

27. 27. A. Parker, L. Vaux, A.M. Patterson, A. Modasia, D. Muraro, A.G. Fletcher, et al., Elevated apoptosis impairs epithelial cell turnover and shortens villi in TNF-driven intestinal inflammation, Cell Death Dis. 10 (2) (2019) 108. https://doi: 10.1038/s41419-018-1275-5.

28. 28. Liang D, Zhuo Y, Guo Z, He L, Wang X, He Y, et al., SIRT1/PGC-1 pathway activation triggers autophagy/mitophagy and attenuates oxidative damage in intestinal epithelial cells, Biochimie 170 (2020) 10-20. https://doi: 10.1016/j.biochi.2019.12.001.

29. 29. Liang H, Liu N, Wang R, Zhang Y, Chen J, Dai Z, et al., N-Acetyl serotonin alleviates oxidative damage by activating nuclear factor erythroid 2-related factor 2 signaling in porcine enterocytes, Antioxidants 9 (2020) (4) 303. https://doi: 10.3390/antiox9040303.

30. 30. H. Zeng, S.K. Hamlin, B.D. Safratowich, W.H. Cheng, L.K. Johnson, Superior inhibitory efficacy of butyrate over propionate and acetate against human colon cancer cell proliferation via cell cycle 
arrest and apoptosis: linking dietary fiber to cancer prevention, Nutr. Res. 83 (2020) 63-72. https://doi: 10.1016/j.nutres.2020.08.009.

31. 31. A. Galano, D.X. Tan, R.J. Reiter, Melatonin: a versatile protector against oxidative DNA damage, Molecules 23 (2018) 530. https://doi: 10.3390/molecules23030530.

32. 32. J.C. Mayo, R.M. Sainz, P. Gonzalez-Menendez, D. Hevia, R. Cernuda, Melatonin transport into mitochondria, Cell Mol. Life Sci. 74 (2017) 3927-3940. https://doi: 10.1007/s00018-017-2616-8.

33. 33. Y.W. Lin, T.Y. Chen, C.Y. Hung, S.H. Tai, S.Y. Huang, C.C. Chang, et al., Melatonin protects brain against ischemia/reperfusion injury by attenuating endoplasmic reticulum stress, Int. J. Mol. Med. 42 (1) (2018) 182-192. https://doi: 10.3892/ijmm.2018.3607.

34. 34. R.J. Reiter, D.X. Tan, J.C. Mayo, R.M. Sainz, J. Leon, D. Bandyopadhyay, Neurally-mediated and neurally-independent beneficial actions of melatonin in the gastrointestinal tract, J. Physiol. Pharmacol. 54 (Suppl 4) (2003) 113125.http://www.jpp.krakow.pl/journal/archive/12_03_s4/articles/10_article.html

35. 35. B. San-Miguel, I. Crespo, D.I. Sanchez, B. González-Fernández, J.J. Ortiz de Urbina, M.J. Tuñón, et al., Melatonin inhibits autophagy and endoplasmic reticulum stress in mice with carbon tetrachloride-induced fibrosis, J. Pineal Res. 59 (2) (2015) 151-162. https://doi: 10.1111/jpi.12247.

36. 36. J. Chen, L. Wang, C. Wu, Q. Hu, C. Gu, F. Yan, et al., Melatonin-enhanced autophagy protects against neural apoptosis via a mitochondrial pathway in early brain injury following a subarachnoid hemorrhage, J. Pineal Res. 56 (1) (2014) 12-19. https://doi: 10.1111/jpi.12086.

37. 37. T. Brzozowski, P.C. Konturek, S.J. Konturek, R. Pajdo, W. Bielanski, I. Brzozowska, et al., The role of melatonin and L-tryptophan in prevention of acute gastric lesions induced by stress, ethanol, ischemia and aspirin, J. Pineal Res. 23 (2) (1997) 79-89. https://doi: 10.1111/j.1600079x.1997.tb00339.x.

38. 38. C.A. De La Lastra, J. Cabeza, V. Motilva, M.J. Martin, Melatonin protects against gastric ischemiareperfusion injury in rats, J. Pineal Res. 23 (2) (1997) 47-52. https://doi: 10.1111/j.1600079x.1997.tb00334.x.

39. 39. T.J. Borody, A. Khoruts, Fecal microbiota transplantation and emerging applications, Nat. Rev. Gastroenterol. Hepatol. 9 (2) (2011) 88-96. https://doi: 10.1038/nrgastro.2011.244.

40. 40. L.P. Smits, K.E. Bouter, W.M. de Vos, T.J. Borody, M. Nieuwdorp, Therapeutic potential of fecal microbiota transplantation, Gastroenterology 145 (5) (2013) 946-953. https://doi: 10.1053/j.gastro.2013.08.058.

41. 41. J.R. Allegretti, Z. Kassam, M. Osman, S. Budree, M. Fischer, C.R. Kelly, The 5D framework: a clinical primer for fecal microbiota transplantation to treat Clostridium difficile infection, Gastrointest. Endosc. 87 (1) (2018) 18-29. https://doi: 10.1016/j.gie.2017.05.036.

42. 42. M. Ghareghani, R.J. Reiter, K. Zibara, N. Farhadi, Latitude, vitamin D, melatonin, and gut microbiota act in concert to initiate multiple sclerosis: A new mechanistic pathway, Front. Immunol. 9 (2018) 2484. https://doi: 10.3389/fimmu.2018.02484. 
43. 43. Y. Jing, D. Yang, F. Bai, C. Zhang, C. Qin, D. Li, et al., Melatonin treatment alleviates spinal cord injury-induced gut dysbiosis in mice, J. Neurotrauma 36 (18) (2019) 2646-2664. https://doi: 10.1089/neu.2018.6012.

44. 44. J. Yin, Y. Li, H. Han, J. Ma, G. Liu, X. Wu, et al., Administration of exogenous melatonin improves the diurnal rhythms of the gut microbiota in mice fed a high-fat diet, mSystems 5 (3) (2020) e0000220. https://doi: 10.1128/mSystems.00002-20.

45. 45. A. Yildirim, S. Arabacı Tamer, D. Sahin, F. Bagriacik, M.M. Kahraman, N.D. Onur, et al., The effects of antibiotics and melatonin on hepato-intestinal inflammation and gut microbial dysbiosis induced by a short-term high-fat diet consumption in rats, Br. J. Nutr. 122 (8) (2019) 841-855. https://doi: 10.1017/S0007114519001466.

46. 46. F. Hong, S. Pan, P. Xu, T. Xue, J. Wang, Y. Guo, et al., Melatonin orchestrates lipid homeostasis through the hepatointestinal circadian clock and microbiota during constant light exposure, Cells 9 (2) (2020) 489. https://doi: 10.3390/cells9020489.

47. 47. S. Yue, D. Zhao, C. Peng, C. Tan, Q. Wang, J. Gong, Effects of theabrownin on serum metabolites and gut microbiome in rats with a high-sugar diet, Food Funct. 10 (11) (2019) 7063-7080. https://doi: 10.1039/c9fo01334b.

48. 48. Bubenik GA, Pang SF, Cockshut JR, Smith PS, Grovum LW, Friendship RM, et al. Circadian variation of portent arterial and venous blood levels of melatonion in pigs and its relationship to food intake and sleep, J. Pineal Research. 28 (1) (2000) 9-15. https://doi: 10.1034/j.1600079x.2000.280102.x.

49. 49. M. Kvietkauskas, V. Zitkute, B. Leber, K. Strupas, P. Stiegler, P. Schemmer, The role of melatonin in colorectal cancer treatment: a comprehensive review, Ther. Adv. Med. Oncol. 12 (2020) 1758835920931714. https://doi: 10.1177/1758835920931714.

50. 50. J.R. Allegretti, B.H. Mullish, C. Kelly, M. Fischer, The evolution of the use of faecal microbiota transplantation and emerging therapeutic indications, Lancet 394 (10196) (2019) 420-431. https://doi: 10.1016/S0140-6736(19)31266-8.

51. 51. S. Kanwal, T.P. Joseph, L. Owusu, R. Xiaomeng, L. Meiqi, X. Yi A, polysaccharide isolated from Dictyophora indusiata promotes recovery from antibiotic-driven intestinal dysbiosis and improves gut epithelial barrier function in a mouse model, Nutrients 10 (8) (2018) 1003. https://doi: 10.3390/nu10081003.

52. 52. S.R. Sammi, J.K. Rawat, N. Raghav, A. Kumar, S. Roy, M. Singh, et al., Galantamine attenuates N,N-dimethyl hydrazine induced neoplastic colon damage by inhibiting acetylcholinesterase and bimodal regulation of nicotinic cholinergic neurotransmission, Eur. J. Pharmacol. 818 (2018) 174183. https://doi: 10.1016/j.ejphar.2017.10.036.

53. 53. M. Tachikawa, K. Amano, K. Nishiyama, A. Urano, K. Kato, K. Yamanaka, Methylamine dichloramine may play a role in the process of colorectal disease through architectural and oxidative changes in crypts in mice, Life Sci. 84 (25-26) (2009) 923-928. https://doi: 10.1016/j.lfs.2009.04.007. 
54. 54. T. Xia, J. Zhang, L. Han, Z. Jin, J. Wang, X. Li, et al., Protective effect of magnolol on oxaliplatininduced intestinal injury in mice, Phytother. Res. 33 (4) (2019) 1161-1172. https://doi: 10.1002/ptr.6311.

55. 55. H. Kim, Y. Jeong, S. Kang, H.J. You, G.E. Ji, Co-culture with Bifidobacterium catenulatum improves the growth, gut colonization, and butyrate production of Faecalibacterium prausnitzii: in vitro and in vivo studies, Microorganisms 8(5) (2020) 788. https://doi: 10.3390/microorganisms8050788.

56. 56. E. Zagato, C. Pozzi, A. Bertocchi, T. Schioppa, F. Saccheri, S. Guglietta, et al., Endogenous murine microbiota member Faecalibaculum rodentium and its human homologue protect from intestinal tumour growth, Nat. Microbiol. 5 (3) (2020) 511-524. https://doi: 10.1038/s41564-019-0649-5.

57. 57. E.C. Soto-Martin, I. Warnke, F.M. Farquharson, M. Christodoulou, G. Horgan, M. Derrien, et al., Vitamin biosynthesis by human gut butyrate-producing bacteria and cross-feeding in synthetic microbial communities, mBio. 11(4) (2020) e00886-20. https://doi: 10.1128/mBio.00886-20.

58. 58. T. Satoh, M. Todoroki, K. Kobayashi, Y. Niimura, S. Kawasaki, Purified thioredoxin reductase from $\mathrm{O}_{2}$-sensitive Bifidobacterium bifidum degrades $\mathrm{H}_{2} \mathrm{O}_{2}$ by interacting with alkyl hydroperoxide reductase, Anaerobe 57 (2019) 45-54. https://doi: 10.1016/j.anaerobe.2019.03.012.

59. 59. G.R. Swanson, A. Gorenz, M. Shaikh, V. Desai, C. Forsyth, L. Fogg, et al. Decreased melatonin secretion is associated with increased intestinal permeability and marker of endotoxemia in alcoholics, Am. J. Physiol. Gastrointest. Liver Physiol. 308 (12) (2015) G1004-G1011. https://doi: 10.1152/ajpgi.00002.2015.

60. 60. G.R. Swanson, J. Siskin, A. Gorenz, M. Shaikh, S. Raeisi, L. Fogg, et al., Disrupted diurnal oscillation of gut-derived short chain fatty acids in shift workers drinking alcohol: Possible mechanism for loss of resiliency of intestinal barrier in disrupted circadian host, Transl. Res. 221 (2020) 97-109. https://doi: 10.1016/j.trsl.2020.04.004.

\section{Figures}



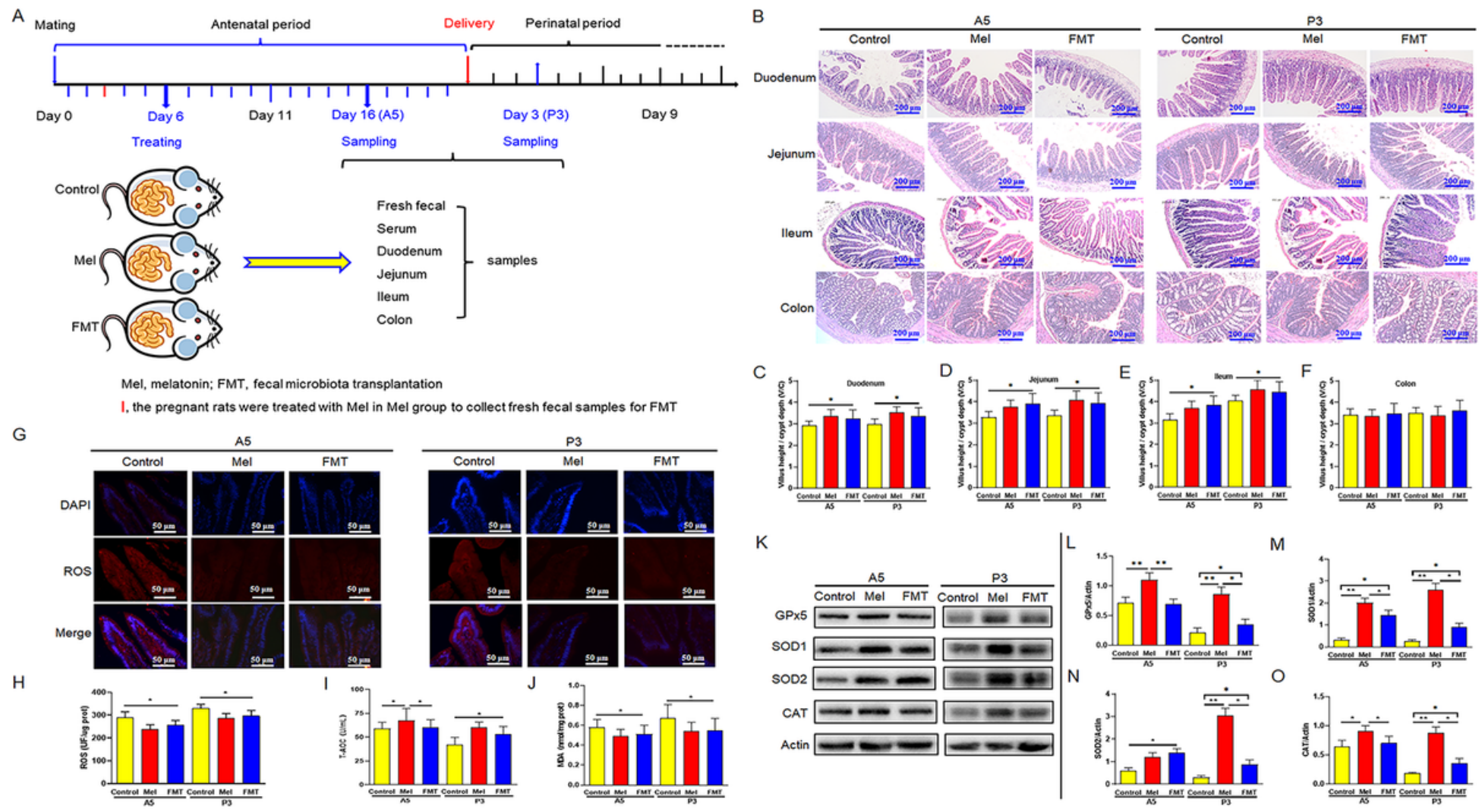
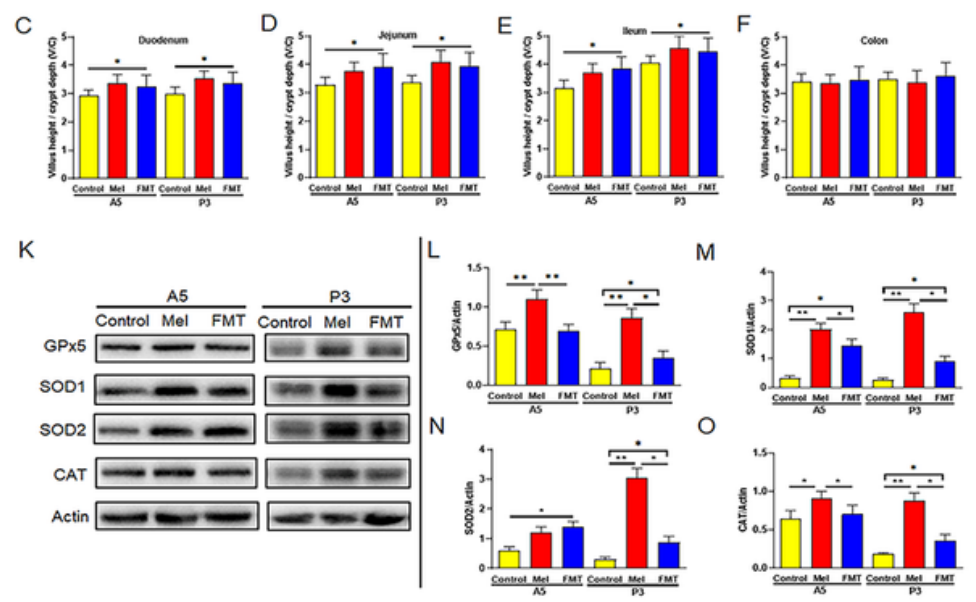

\section{Figure 1}

Melatonin and its-remodeled FMT increase intestinal villus height/crypt depth (V/C) by enhancing antioxidation in antenatal and perinatal female rats. The timeline of treatments and sample collection in this study $(A)$. Intestinal histological morphology indicated by H\&E staining in A5 and P3 rats $(n=5$ rats/group) (B). The ratio of intestinal villus height/crypt depth of duodenum (C), jejunum (D), ileum (E) and colon $(\mathrm{F})$. A minimum of 10 tissue sections were cut from each sample. Five longest villi in each tissue section and a total of 250 longest villi were analyzed in each group. lleum histological immunofluorescent imagines showed ROS level after treated with melatonin and FMT in A5 and P3 rats (G). The detections of ROS (H), T-AOC (I) and MDA in intestinal serum (J). Intestinal tissue expression of GPX5, SOD1, SOD2 and CAT proteins $(n=3)(K)$. The protein level analysis of GPX5 (L), SOD1 (M), SOD2 $(\mathrm{N})$ and CAT (O). Mel, melatonin; FMT, fecal microbiota transplantation; A5, antenatal female rat on the day 5. P3, perinatal female rat on the day $3 .{ }^{*} \mathrm{P}<0.05$, ${ }^{\star *} \mathrm{P}<0.01$. 
A

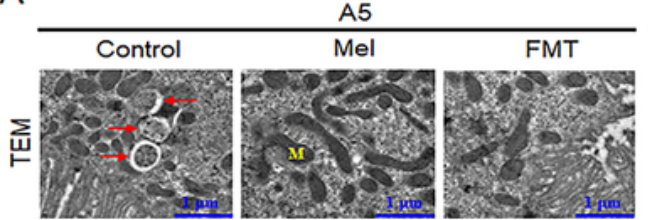

C

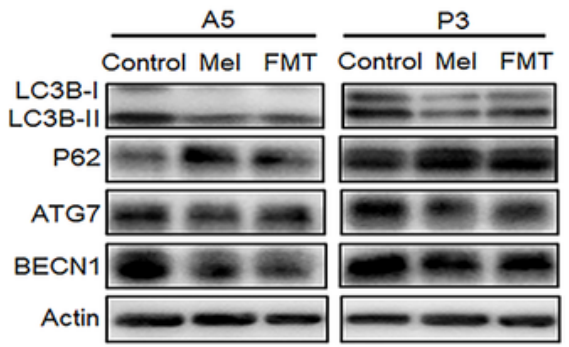

D

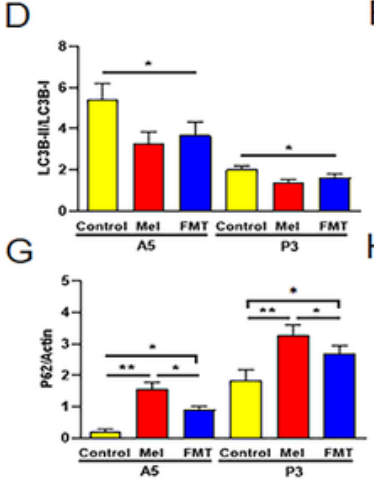

B

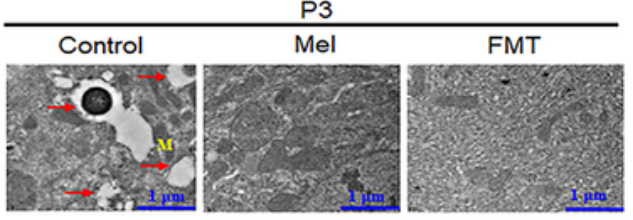

E

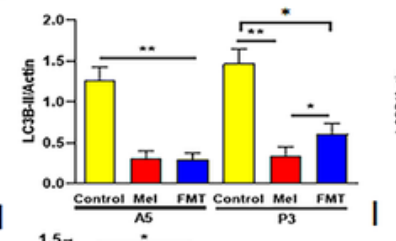

F
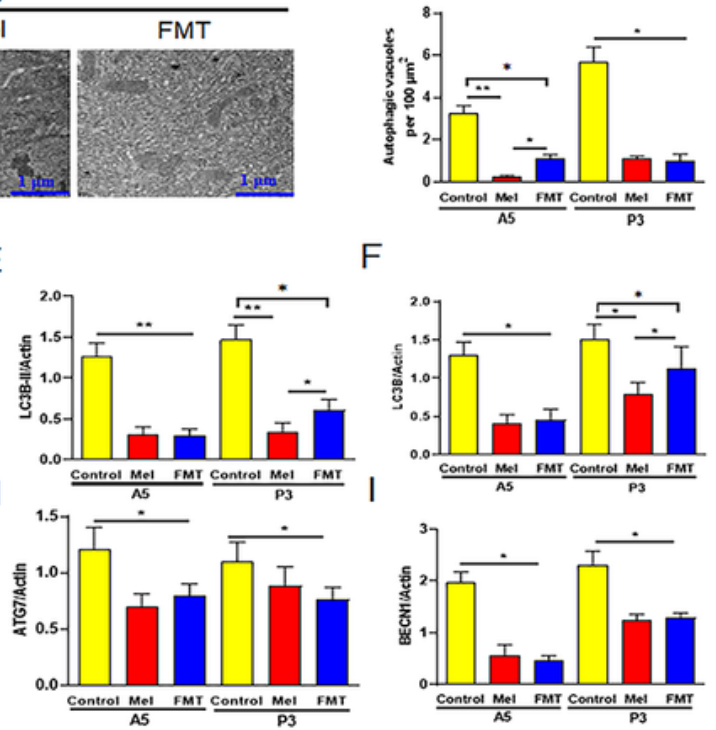
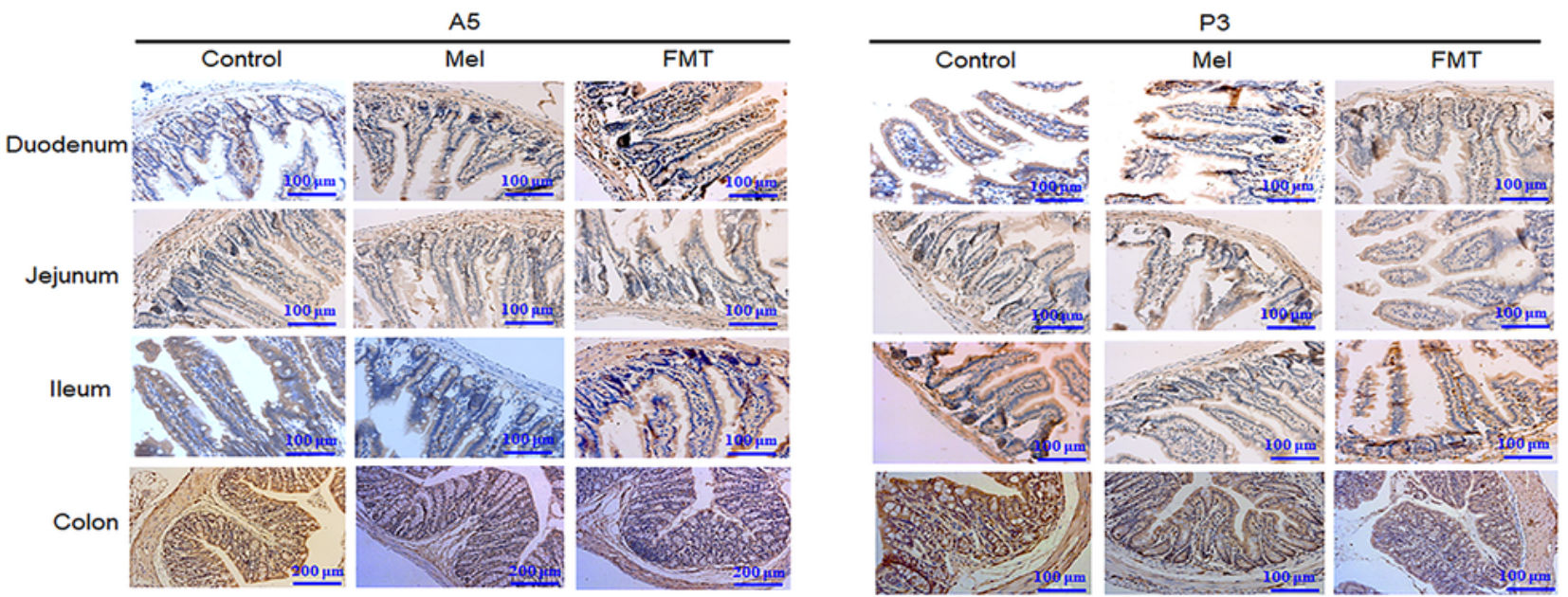

K

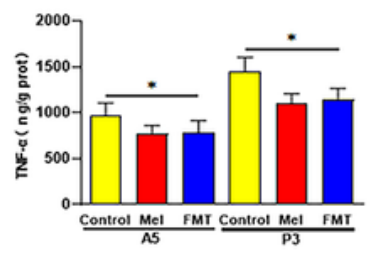

O

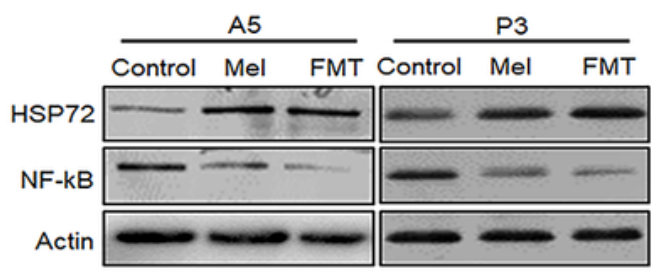

L

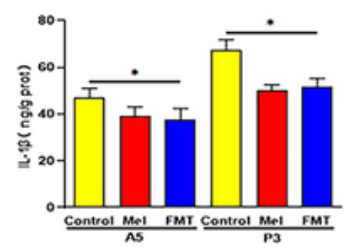

P
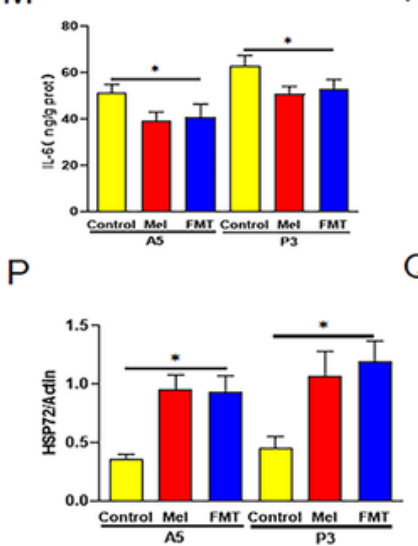

N

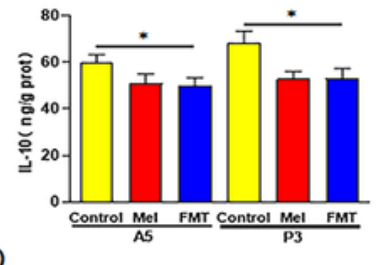

Q

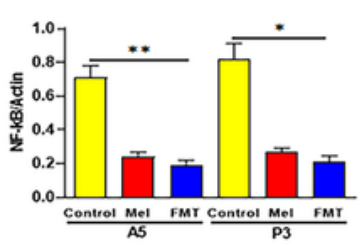

\section{Figure 2}

Melatonin and FMT inhibit gut autophagy and inflammation in antenatal and perinatal female rats. Intestinal histological images using transmission electron microscopy (TEM) in A5 and P3 rats. Red arrows indicate autophagic vacuole, and $\mathrm{M}$ indicates mitochondrion (A). Analysis of autophagic vacuoles in the intestinal epithelial cells. For counting autophagic vacuoles including autophagosomes and autolysosomes, 10 randomly selected images in each sample and data from 5 rats in each group were 
compared (B). Intestinal tissue expression of LC3B-I, LC3B-II, P62, ATG7 and BECN1 proteins $(\mathrm{n}=3)(\mathrm{C})$. The protein level analysis of LC3B-II/LC3B-I (D), LC3B-I (E), LC3B-II (F), P62 (G), ATG7 (H) and BECN1 (I). Intestinal immunohistochemical images using antibody NF-KB (J). Detections of TNF-a (K), IL-1 $\beta(L)$, IL-6 $(M)$ and IL-10 $(N)$ in serum $(n=5)$. intestinal tissue expression of HSP72 and NF-KB proteins $(n=3)(0)$. The protein levels of HSP72 (P) and NF-Kb (Q). *P $<0.05$, ** $\mathrm{P}<0.01$.

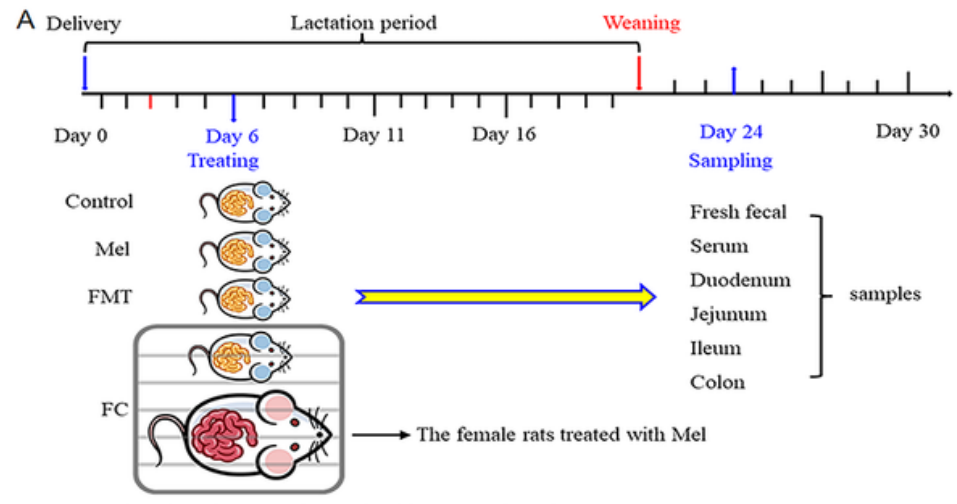

Mel, melatonin; FMT, fecal microbiota transplantation; FC, Foster care

I, the suckling rats were treated with Mel in Mel group to collect fresh fecal samples for FMT

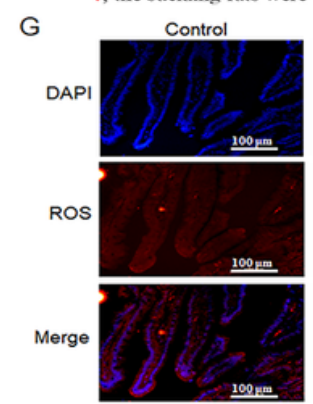

$\mathrm{H}$

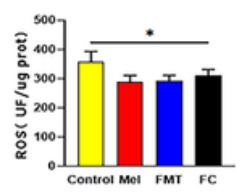

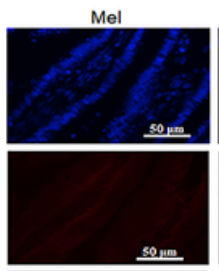
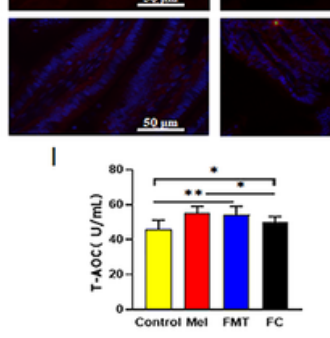
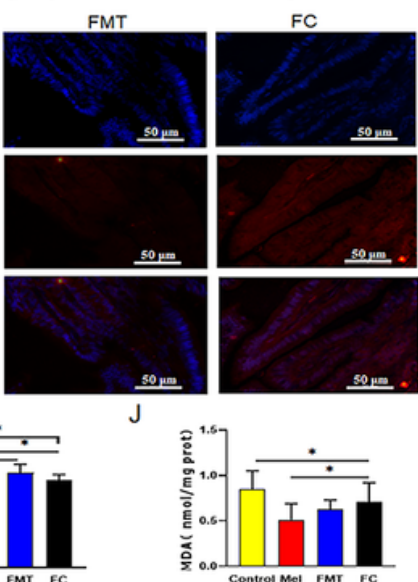

B
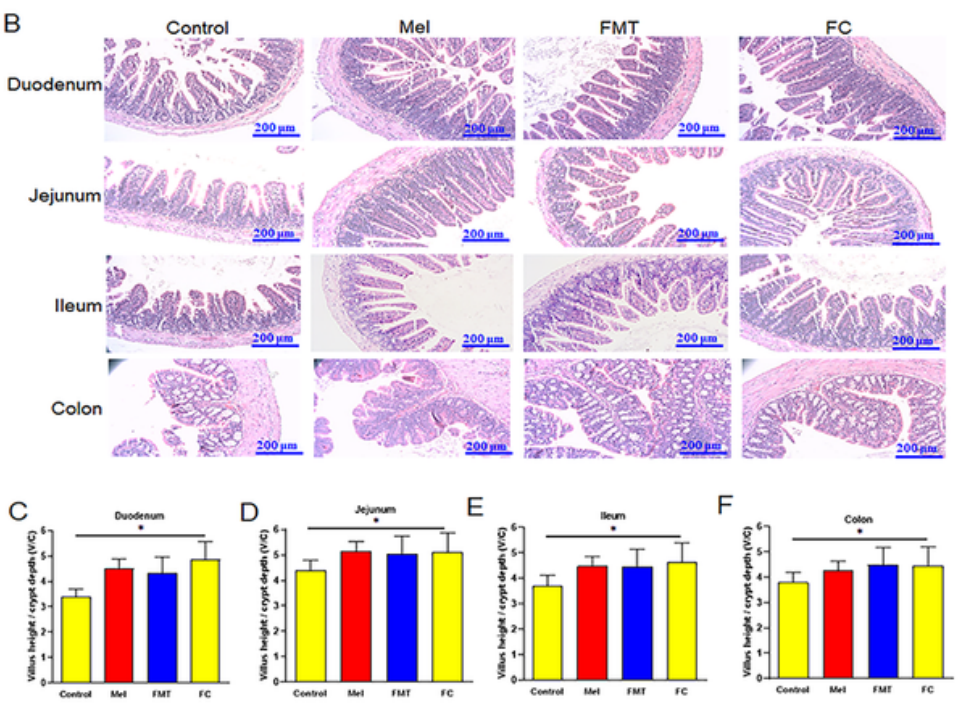

K
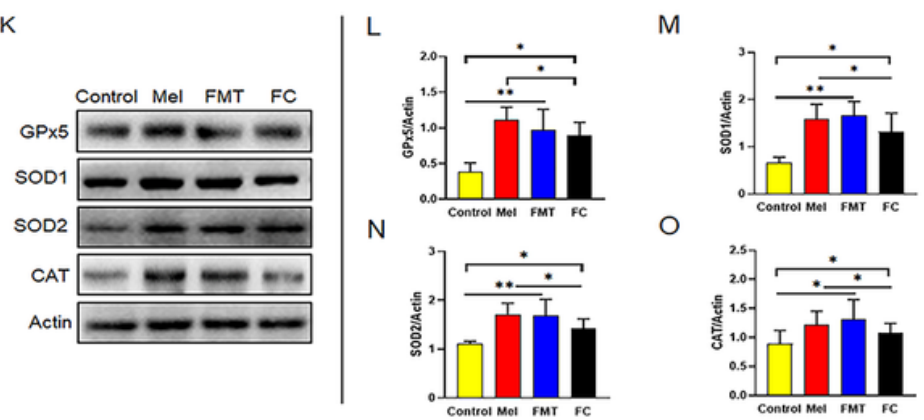

$\mathrm{O}$

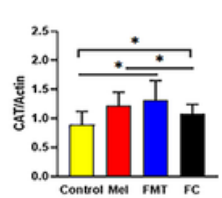

\section{Figure 3}

Melatonin, FMT and FC improve intestinal V/C through inhibiting oxidative stress in early weaned rat. The timeline of treatments and sample collection (A). Intestinal histological morphology indicated by H\&E staining in early weaned rats on the day 3 after weaning ( $n=20$ rats/group) (B). The $V / C$ ratio of duodenum (C), jejunum (D), ileum (E) and colon (F). A minimum of 10 tissue sections were cut from each sample (randomly selected 10 rats/group). Five longest villi in each tissue section and a total of 500 longest villi were analyzed in each group. Ileum histological immunofluorescent imagines showed ROS level after treated with melatonin, FMT and FC in early weaned rats (G). The detections of ROS (H), T-AOC $(\mathrm{I})$ and MDA $(\mathrm{J})$ in intestinal tissue or serum $(n=10)$. Intestinal tissue expression of GPX5, SOD1, SOD2 and CAT proteins $(n=3)(K)$. The protein levels of GPX5 $(L)$, SOD1 $(M)$, SOD2 $(N)$ and CAT $(0)$. FC, foster care. ${ }^{*} \mathrm{P}<0.05, * * \mathrm{P}<0.01$. 
A

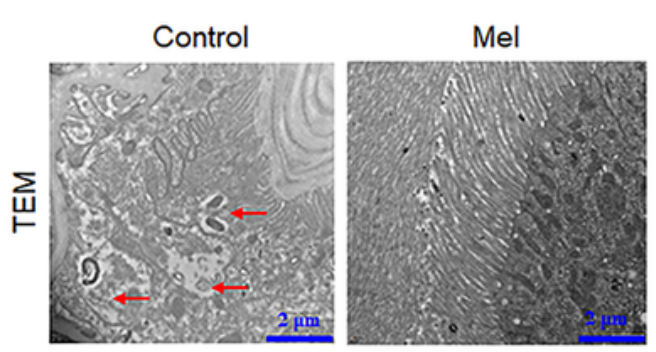

C

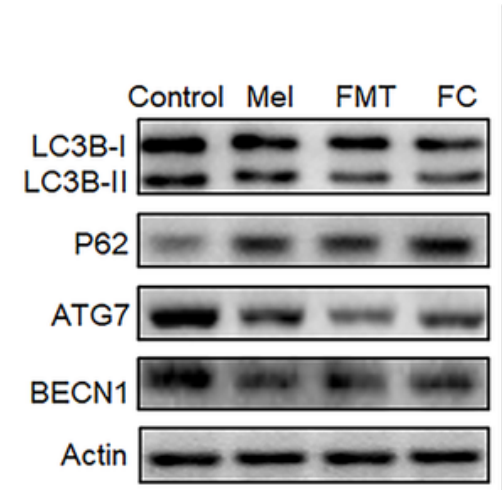

D

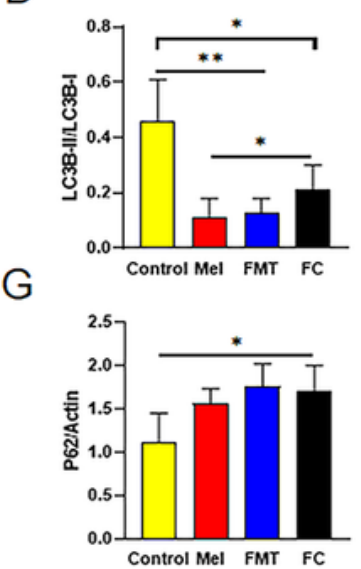

J

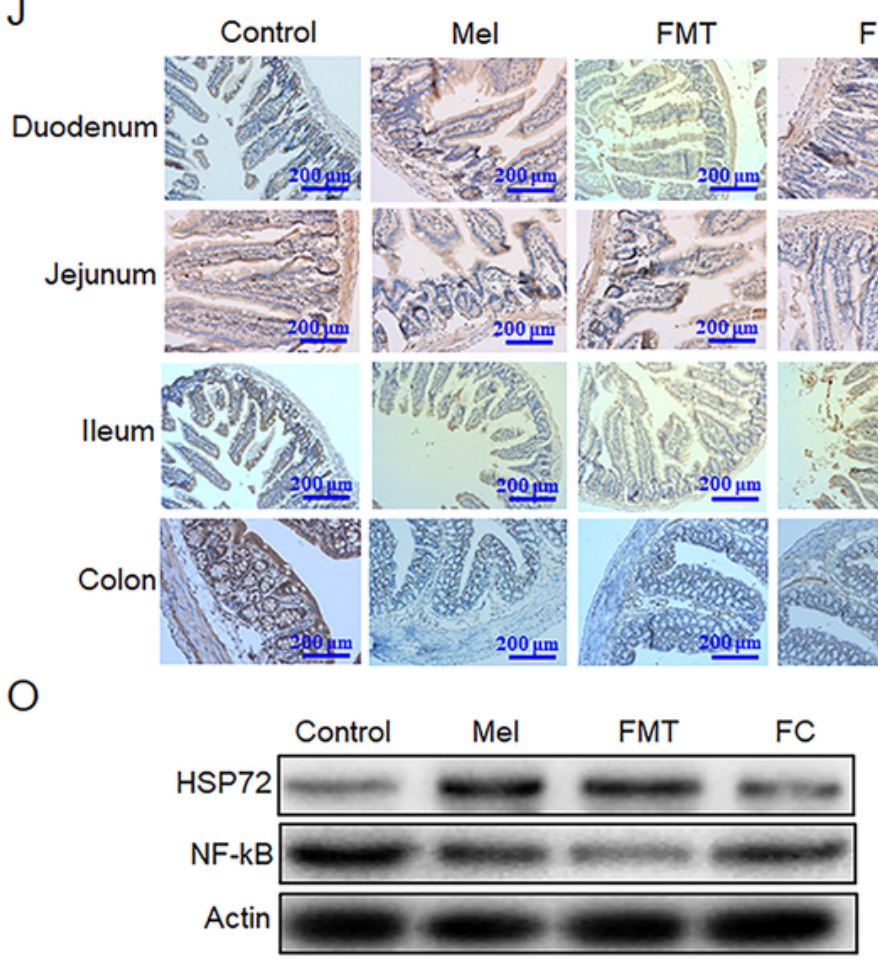

FMT

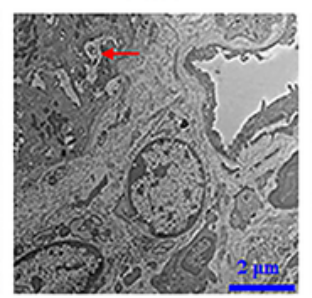

E

$\mathrm{H}$
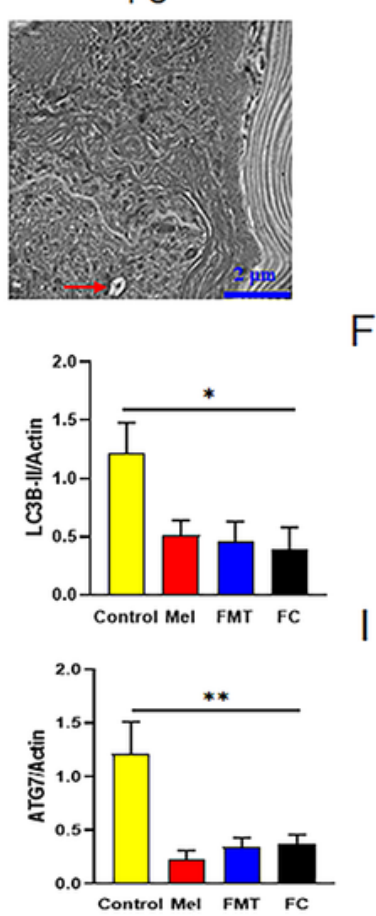

K

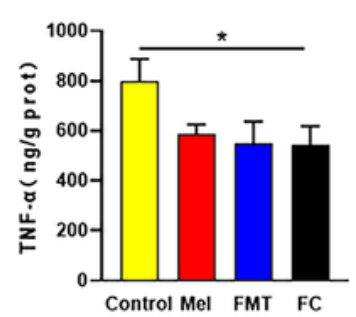

$M$

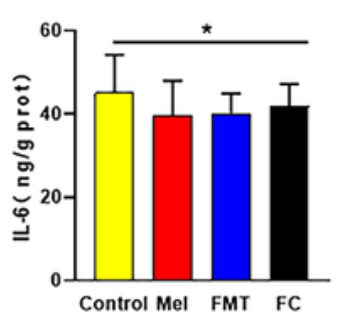

P

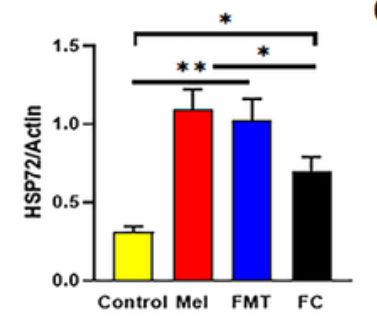

B

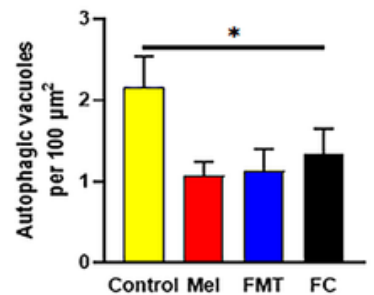

F
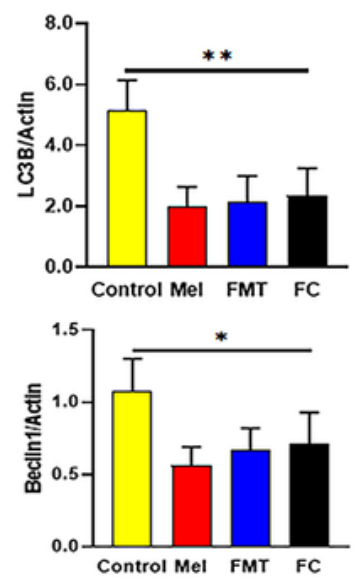

L

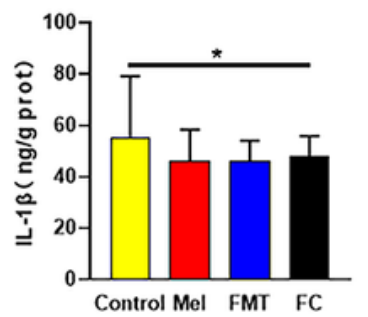

$\mathrm{N}$

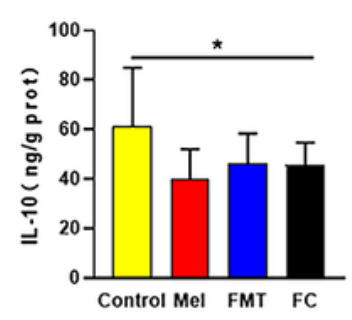

Q

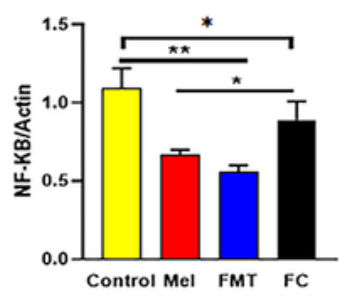

Figure 4

Melatonin, FMT and FC suppress gut autophagy and inflammation in early weaned rats. Intestinal histological images using TEM and red arrows indicate autophagic vacuoles (A). Analysis of autophagic vacuoles in intestinal cells. For counting autophagic vacuoles, 5 randomly selected images in each sample and data from 10 rats in each group were compared (B). Intestinal tissue expression of LC3B-I, LC3B-II, P62, ATG7 and BECN1 proteins $(n=3)(C)$. The protein levels of LC3B-II/LC3B-I (D), LC3B-I (E), 
LC3B-II (F), P62 (G), ATG7 (H) and BECN1 (I). (J) Intestinal immunohistochemical images using antibody NF-KB. The detections of TNF-a (K), IL-1 $\beta(L), I L-6(M)$ and IL-10 $(N)$ in serum. $(n=10)$. Intestinal tissue expression of HSP72 and NF-KB proteins $(n=3)(0)$. The protein levels of HSP72 (P) and NF-KB $(Q)$, *P< $0.05, * * \mathrm{P}<0.01$.

A

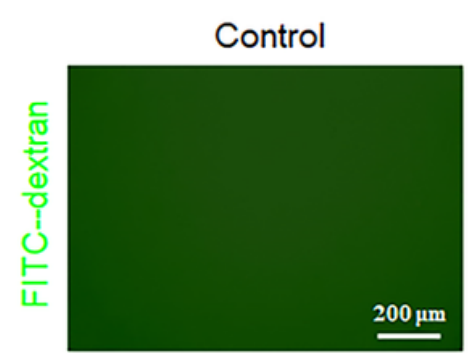

B

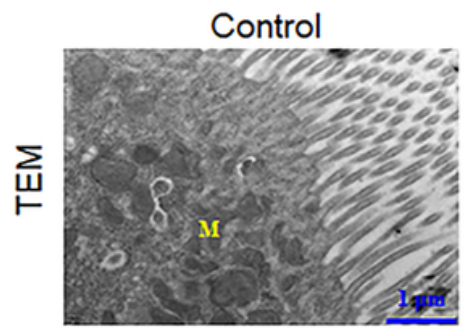

C

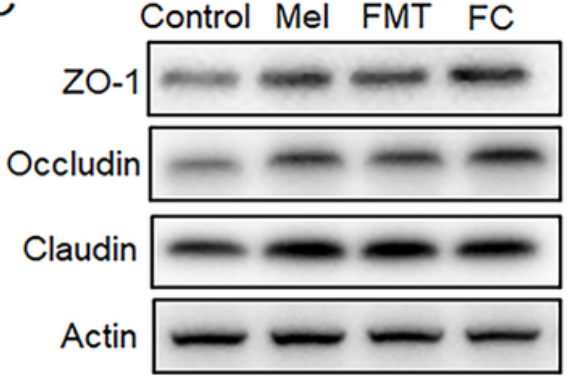

Mel

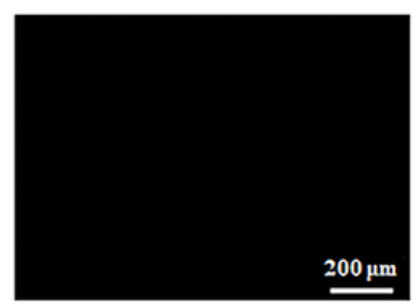

Mel

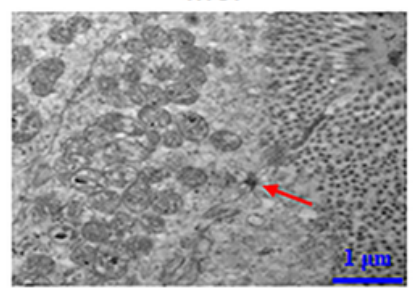

D

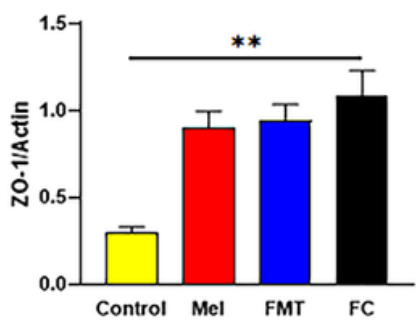

FMT

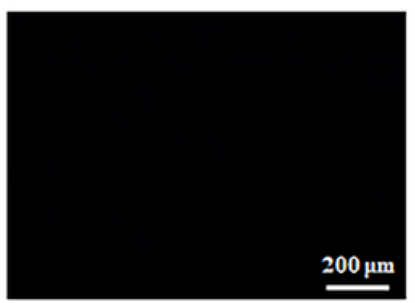

FMT

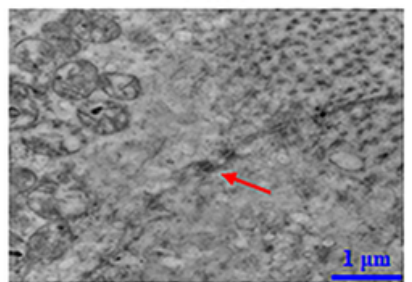

$E$

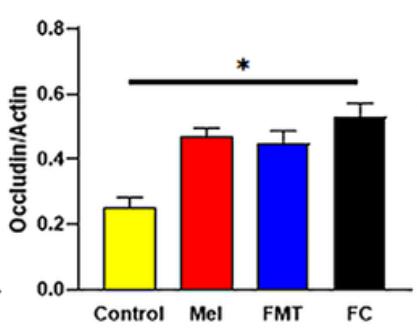

FC

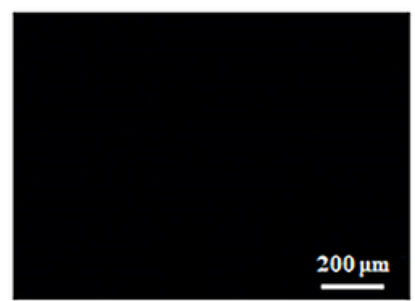

FC

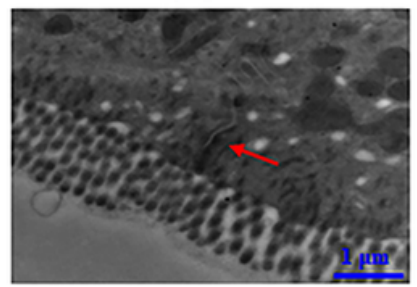

F

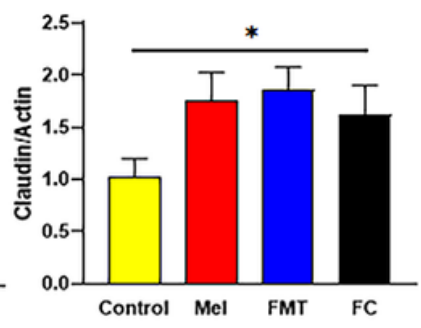

\section{Figure 5}

Melatonin, FMT and FC decrease intestinal permeability in early weaned rats. Serum FITC staining $(\mathrm{n}=$ 10) (A). Intestinal histological images using TEM. The red arrow indicates tight junction protein. $M$, mitochondrion (B). Intestinal tissue expression of ZO-1, Occludin and Claudin proteins $(n=3)(C)$. The protein levels of ZO-1 (D), Occludin (E) and Claudin (F). ${ }^{*} P<0.05, * * P<0.01$. 


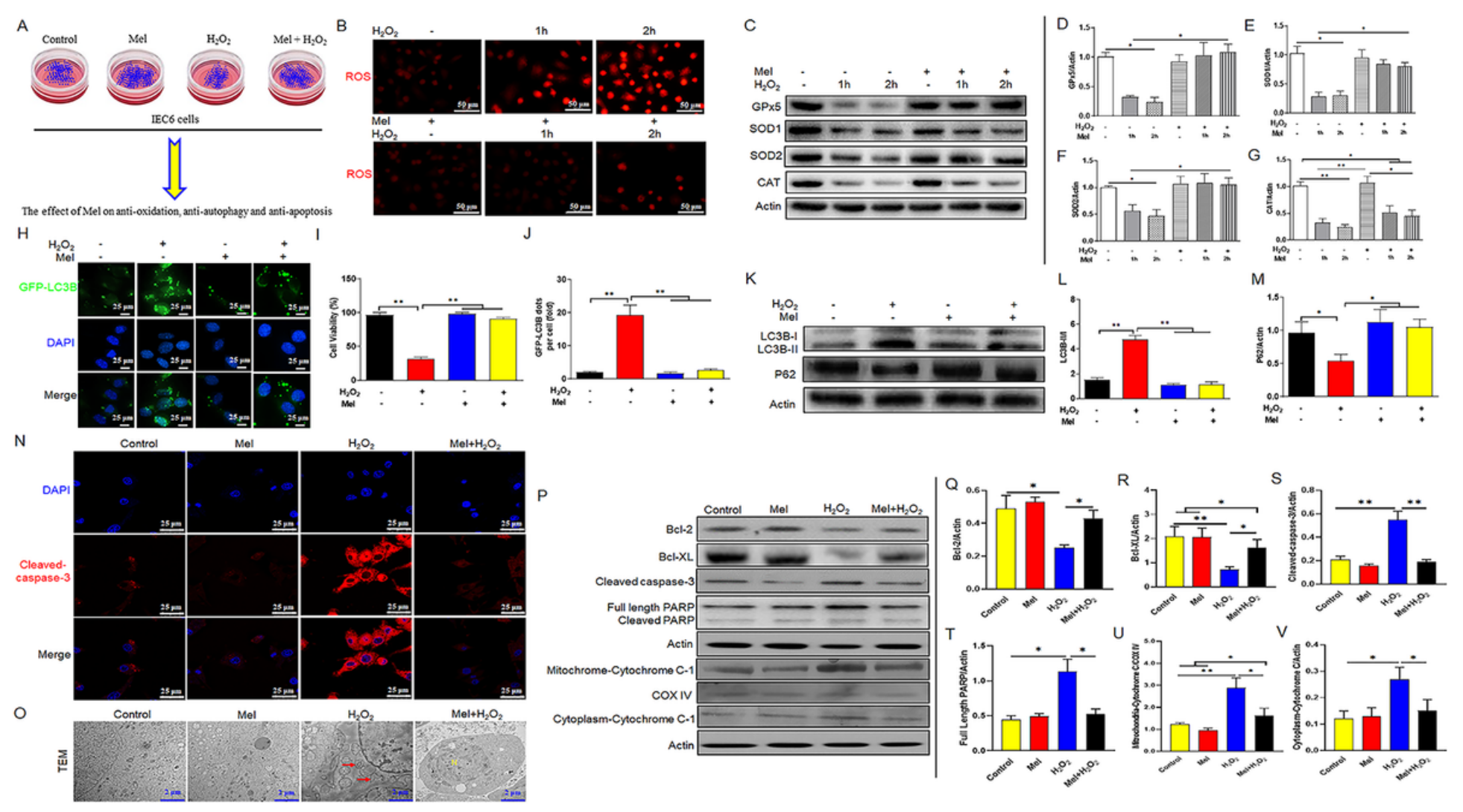

Figure 6

Melatonin enhances antioxidation to attenuate autophagy and apoptosis in IEC6 treated with $\mathrm{H} 2 \mathrm{O} 2$. The cells were pretreated with $10 \mu \mathrm{M}$ melatonin for 24 hours, followed by $200 \mu \mathrm{M} \mathrm{H} 2 \mathrm{O} 2$ stimulation for 1 or 2 hours before they were used for the next assay. Experiment design for the effect of Mel on anti-oxidation, anti-autophagy and anti-apoptosis in IEC6 cells (A). Melatonin was against oxidation in IEC6 treated with H2O2 (B). Cell expression of LC3B-I, LC3B-II, P62, ATG7, BECN1, GPX5, SOD1, SOD2 and CAT proteins ( $n=$ 3) (C). The protein levels of above proteins (D-G). Melatonin attenuates autophagy in IEC6 treated with $\mathrm{H} 2 \mathrm{O} 2(\mathrm{H})$. Cell viability analysis $(n=6)(\mathrm{I})$. Analysis of GFP-LC3B dots per cells $(\mathrm{J})$. Cell expression of LC3B-I, LC3B-II and P62 proteins $(n=3)(K)$. The protein levels of LC3B-II/LC3B-I (L) and P62 (M). Relative fluorescence intensity of Cleaved-caspase 3 in the treatment of melatonin with or without $\mathrm{H} 2 \mathrm{O} 2(\mathrm{~N})$. TEM images of cell ultrastructure of IEC- 6 cells treated with Mel, $\mathrm{H} 2 \mathrm{O} 2$ and Mel plus $\mathrm{H} 2 \mathrm{O} 2$. The red arrow indicates apoptotic body. N, cell nucleus (0). Cell expression of Cleaved-caspase-3, Bcl-2, Bcl-XL, full length PARP, cleaved PARP, mitochrome-Cytochrome $\mathrm{C}-1$ and cytoplasm-Cytochrome $\mathrm{C}-1$ proteins $(\mathrm{n}=3)$ (P). (Q-V) The protein levels of above genes. ${ }^{*} \mathrm{P}<0.05,{ }^{*} \mathrm{P}<0.01$. 
A

$\begin{array}{lcccccc}\begin{array}{l}\text { Control } \\ (n=5)\end{array} & \begin{array}{c}\text { Mel } \\ (n=5)\end{array} & \begin{array}{c}\text { FMT } \\ (n=5)\end{array} & \begin{array}{l}\text { Control } \\ (n=5)\end{array} & \begin{array}{c}\text { Mel } \\ (n=5)\end{array} & \begin{array}{c}\text { FMT } \\ (n=5)\end{array} & \begin{array}{l}\text { Control Mel FMT } \\ (n=5)\end{array}(n=5) \quad(n=5)\end{array}$

$(n=5)(n=5) \quad(n=5) \quad(n=5)$

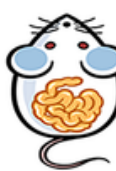
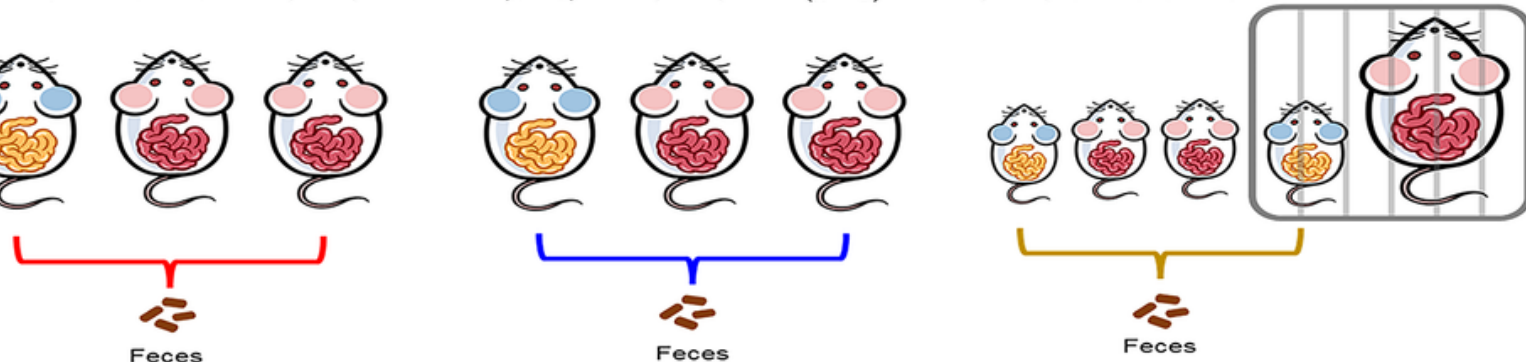

Antenatal day 5

L

Perinatal day 3

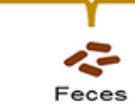

Weaning day 3
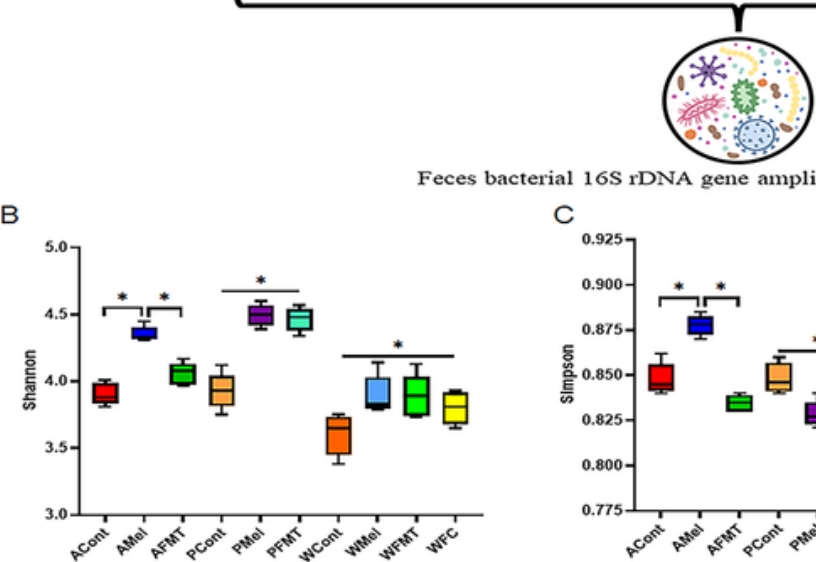

Feces bacterial $16 \mathrm{~S}$ rDNA gene amplicon sequencing analysis
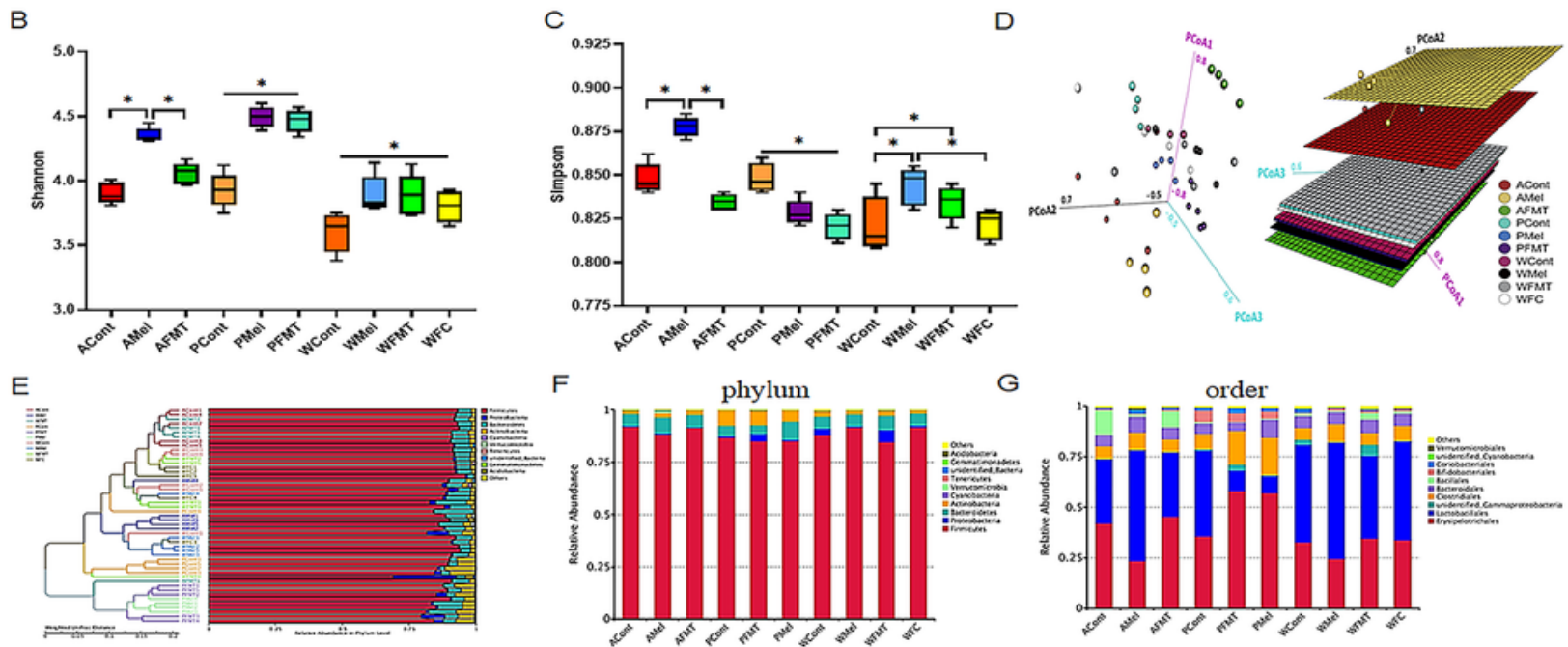

$\mathrm{H}$

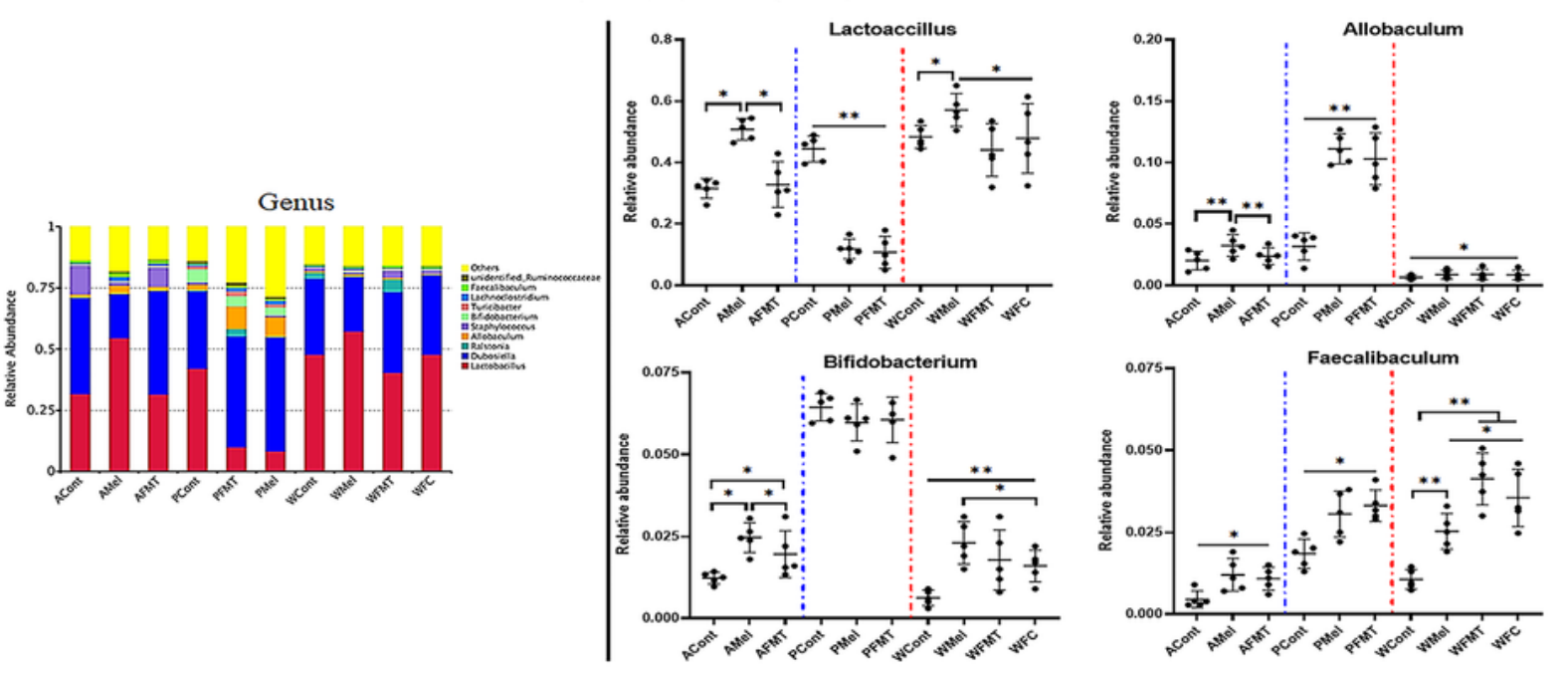

Figure 7

Melatonin, FMT and FC increase the abundances of Allobaculum, Bifidobacterium and Faecalibaculum by remodeling gut microbiota in rats. Experiment design for feces bacterial 16S rDNA gene amplicon sequencing analysis (A). Shannon index in a-diversity analysis (B). Simpson index in a-diversity analysis (C). PCoA plot analysis from each sample $(n=5)(D)$. weighted UniFrac distance in each sample $(E)$. Microbiota compositions and abundances at the phylum (F), order (G) and genus (H) levels, respectively. 
ACont, antenatal control. AMel, antenatal melatonin. AFMT, antenatal FMT. PCont, perinatal control. PMel, perinatal melatonin. PFMT, perinatal FMT. WCont, weaned control. WMel, weaned melatonin. WFMT weaned FMT. WFC, weaned foster care. ${ }^{*} \mathrm{P}<0.05,{ }^{*} \mathrm{P}<0.01$.

A

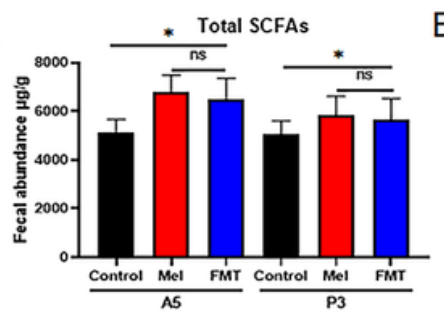

E

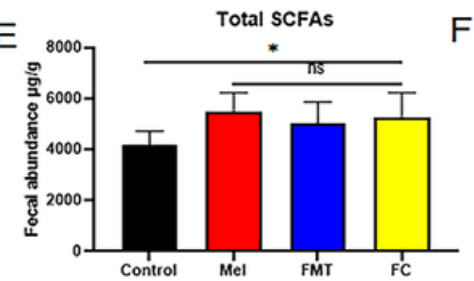

I

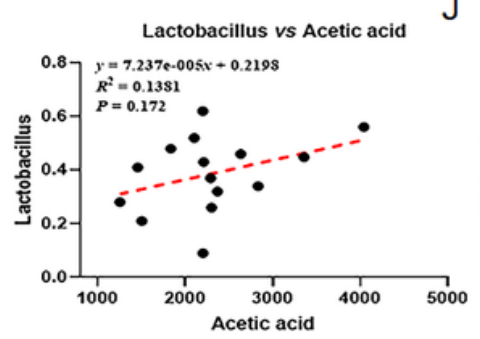

M

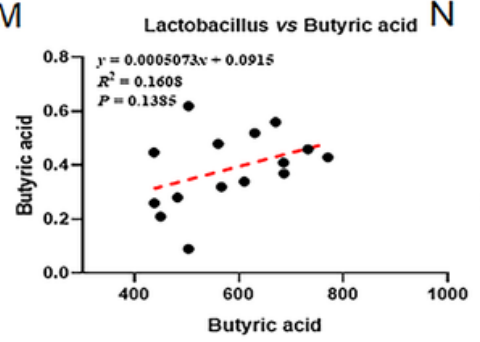

$\mathrm{B}$

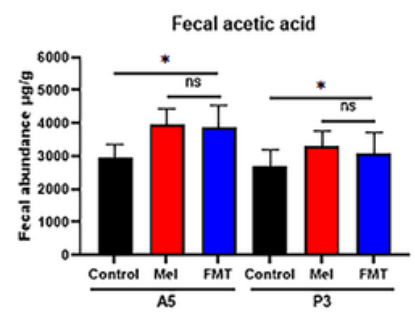

F
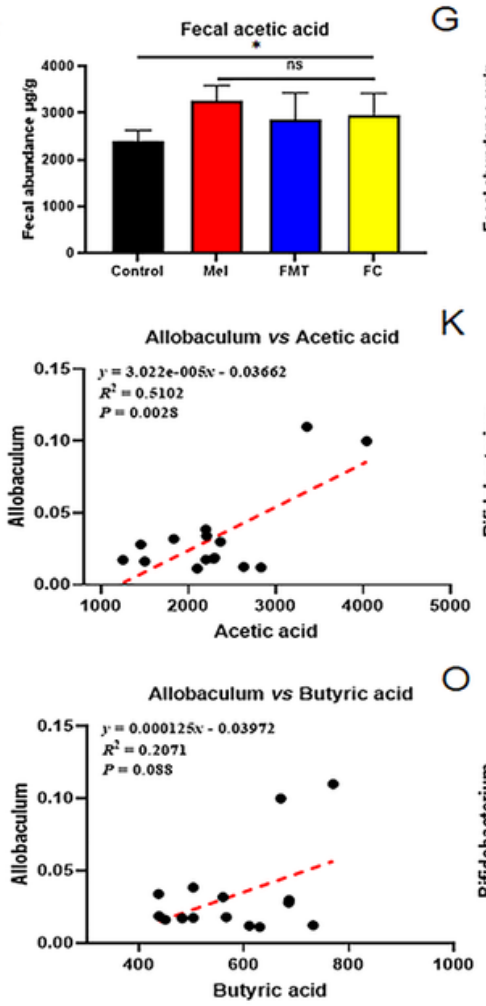

C

G

K
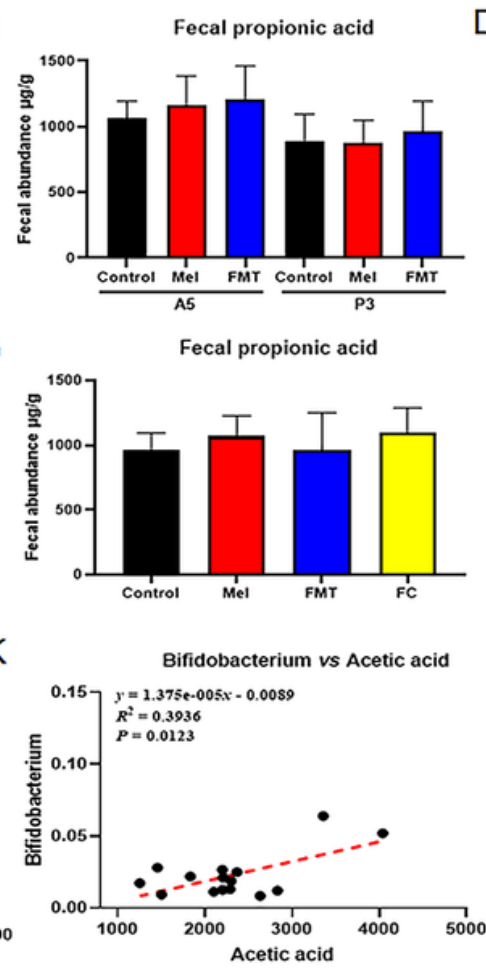

Bifidobacterium vs Butyric acid

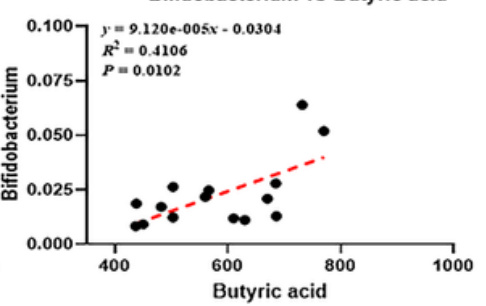

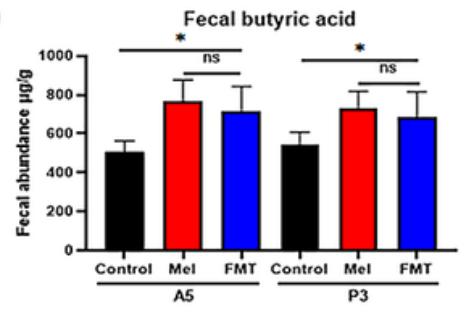

$\mathrm{H}$

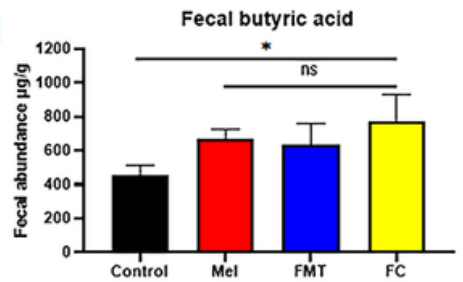

$\mathrm{L}$

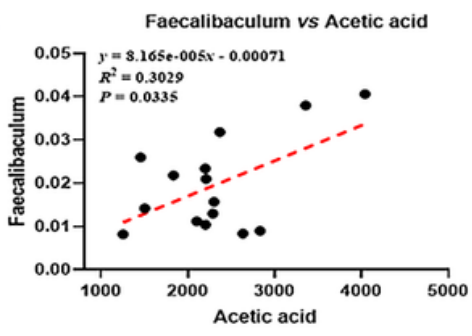

$P$

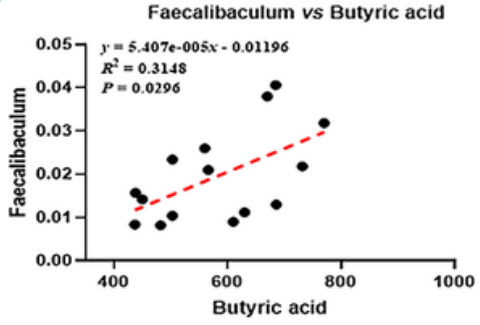

Figure 8

Melatonin, FMT and FC protect intestinal health through more SCFAs produced by reprogrammed gut microbiota. The concentrations of fecal total SCFAs (A), acetic acid (B), propionate (C), and butyrate (D) in antenatal and perinatal female rats. The concentrations of fecal total SCFAs (E), acetic acid (F), propanoic acid $(\mathrm{G})$ and butyric acid $(\mathrm{H})$ in early weaned rats. Correlation analyses between acetic acid and Lactobacillus (I). Correlation analyses between acetic acid and Allobaculum (J). Correlation analyses between acetic acid and Bifidobacterium (K). Correlation analyses between propanoic acid and Faecalibaculum (L). Correlation analyses between butyric acid and Lactobacillus (M). Correlation analyses between butyric acid and Allobaculum $(\mathrm{N})$. Correlation analyses between butyric acid and Bifidobacterium (0). Correlation analyses between butyric acid and Faecalibaculum; Fecal SCFAs were determined by gas chromatography. Differences were assessed by Student's $t$ test, ${ }^{*} P<0.05$. Correlation analysis between microbiota and acetic acid was conducted by Pearson correlation analysis $(P)$. 


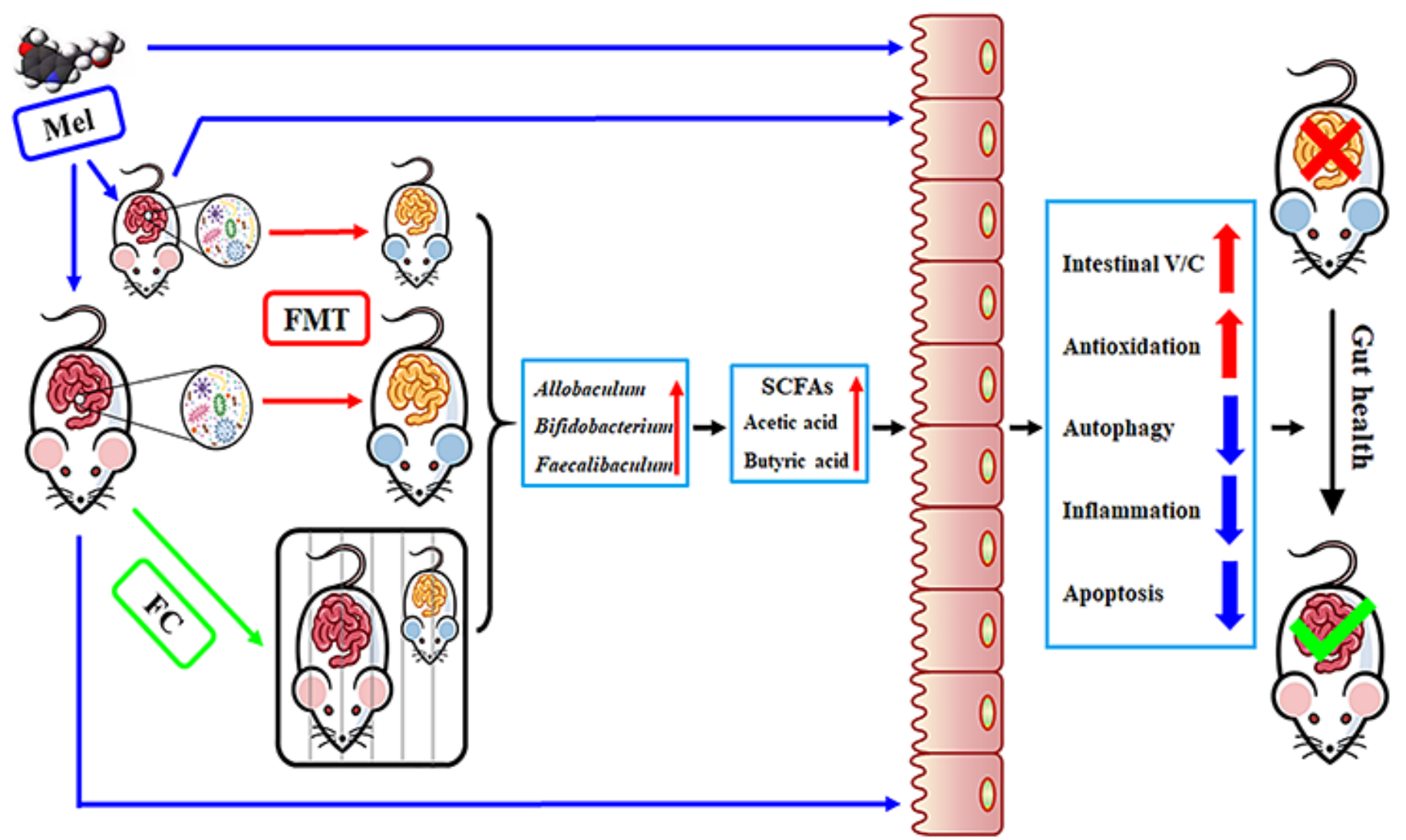

Mel, melatonin; FMT: fecal microbiota transplantation; FC, foster care; SCFAs, short-chain fatty acids; V/C, intestinal villus height/crypt depth

Figure 9

Schematic diagram for melatonin, FMT and FC improving gut health through increasing intestinal V/C, enhancing antioxidation, and inhibiting autophagy, inflammatory and apoptosis.

\section{Supplementary Files}

This is a list of supplementary files associated with this preprint. Click to download.

- Fig.S1.tif

- Fig.S2.tif

- Fig.S3.tif

- Fig.S4.tif

- SupplementaryFile.docx 